DIAS-09-01

HWM-09-01

EMPG-09-01

\title{
Dimensional Reduction, Monopoles and Dynamical Symmetry Breaking
}

\author{
Brian P. Dolan ${ }^{1,2}$ and Richard J. Szabo ${ }^{3}$ \\ ${ }^{1}$ School of Theoretical Physics, Dublin Institute of Advanced Studies \\ 10 Burlington Road, Dublin 4, Ireland \\ ${ }^{2}$ Department of Mathematical Physics, National University of Ireland \\ Maynooth, Co. Kildare, Ireland \\ ${ }^{3}$ Department of Mathematics and Maxwell Institute for Mathematical Sciences \\ Heriot-Watt University, Colin Maclaurin Building, Riccarton, Edinburgh EH14 4AS, U.K. \\ Email: bdolan@thphys.nuim.ie , R.J.Szabo@ma.hw.ac.uk
}

\begin{abstract}
We consider SU(2)-equivariant dimensional reduction of Yang-Mills-Dirac theory on manifolds of the form $M \times \mathbb{C} P^{1}$, with emphasis on the effects of non-trivial magnetic flux on $\mathbb{C} P^{1}$. The reduction of Yang-Mills fields gives a chain of coupled Yang-Mills-Higgs systems on $M$ with a Higgs potential leading to dynamical symmetry breaking, as a consequence of the monopole fields. The reduction of SU(2)-symmetric fermions gives massless Dirac fermions on $M$ transforming under the low-energy gauge group with Yukawa couplings, again as a result of the internal U(1) fluxes. The tower of massive fermionic Kaluza-Klein states also has Yukawa interactions and admits a natural $\mathrm{SU}(2)$-equivariant truncation by replacing $\mathbb{C} P^{1}$ with a fuzzy sphere. In this approach it is possible to obtain exactly massless chiral fermions in the effective field theory with Yukawa interactions, without any further requirements. We work out the spontaneous symmetry breaking patterns and determine the complete physical particle spectrum in a number of explicit examples.
\end{abstract}




\section{Introduction}

Various schemes have been used to suggest that the Higgs and Yukawa sectors of the standard model of particle physics may find their natural origin in a higher-dimensional gauge theory. The natural candidates for compact internal spaces in such Kaluza-Klein models are coset spaces $G / H$, as the action of the isometry group $G$ can be elegantly compensated by gauge transformations in such a way that the Lie derivative with respect to a Killing vector becomes a gauge generator. This provides a unification of the gauge and Higgs sectors in higher dimensions, while the coupling of fermions to the higher-dimensional gauge theory naturally induces Yukawa couplings after dimensional reduction. The pioneering scheme realizing these constructions is called "coset space dimensional reduction" $[1,2]$. It has also been used more recently for the dimensional reduction of ten-dimensional supersymmetric gauge theories to four-dimensional field theories with softly broken $\mathcal{N}=1$ supersymmetry [3], and for the reduction of superstring theories on nearly Kähler manifolds [4]. On the other hand, a generic problem with Kaluza-Klein reductions has been that they are unable to generate chiral gauge theories, without some additional modifications $[2,5]$.

In coset space dimensional reduction, one imposes constraints on the higher-dimensional fields which ensures that they are invariant under the $G$-action up to gauge transformations. They amount to studying embeddings of $G$ or of its closed subgroup $H$ in the gauge group of the higherdimensional theory. The solutions of the constraints are then formally identified with the lowest modes of the Kaluza-Klein towers of the fields, in a field expansion in harmonics on the compact coset space $G / H$. However, this scheme does not seem to naturally allow for the incorporation of topologically non-trivial background fields on $G / H$ which arise from gauging the holonomy subgroup $H$. It has been shown in [6] that, for certain coset spaces, the inclusion of non-trivial internal fluxes can induce the chiral fermionic spectrum of quarks and leptons of the standard model.

In this paper we will study the dimensional reduction of gauge theories in a way which naturally incorporates the topology of gauge fields on $G / H$. To distinguish our approach from the more standard coset space techniques, we will refer to it as "equivariant dimensional reduction". The general formalism is developed in $[7,8]$ and has been used to describe vortices as generalized instantons of higher-dimensional Yang-Mills theory [9]-[14], as well as to construct explicit SU(2)-equivariant monopole and dyon solutions of pure Yang-Mills theory in four dimensions [15]. Although similar in spirit to the coset space dimensional reduction scheme, this approach systematically constructs the unique field configurations on the higher-dimensional space which are equivariant with respect to the internal isometry group $G$ and reduces Yang-Mills theory to a quiver gauge theory. As in coset space dimensional reduction, there is a priori no relation between the gauge group $\mathcal{G}$ of the higher-dimensional field theory and the groups $G$ or $H$, and the resulting gauge group of the dimensionally reduced field theory is a subgroup of $\mathcal{G}$. This is in contrast to the usual Kaluza-Klein reductions where the isometry group (or the holonomy group) is identified with the gauge group.

In the following we analyse in detail the simplest case where $G=\mathrm{SU}(2)$ and $H=\mathrm{U}(1)$, so that the internal space is the projective line $\mathbb{C} P^{1}$. In this case the equivariant dimensional reduction of gauge fields naturally comes with Dirac monopoles. We will emphasize the effects of the non-trivial monopole background on the physical particle spectrum obtained from reduction of a Yang-MillsDirac theory. As usual, the mass scale of the dimensionally reduced field theory is set by the size

of the internal space. We will obtain a Higgs sector of the lower-dimensional gauge theory with a Higgs potential that leads to dynamical symmetry breaking, as a direct consequence of the monopole charges. We work out the complete physical particle content and masses for a variety of symmetry hierarchies, including one that entails the hierarchy $\mathrm{SU}(3) \rightarrow \mathrm{SU}(2) \times \mathrm{U}(1) \rightarrow \mathrm{U}(1)$ in which the second step is dynamical electroweak symmetry breaking. An induced Yukawa sector of the reduced fermionic field theory naturally emerges, again as a direct result of the internal fluxes. Starting 
with massless fermions in higher dimensions, our dimensional reduction induces both massless and massive fermions. In particular, it naturally allows for the reduction to massless chiral fermions without the imposition of any extra structure. In the case of higher spinor harmonic modes, which generate massive fermions, we show that replacing the coset $\mathbb{C} P^{1}$ with a fuzzy sphere gives a natural $\mathrm{SU}(2)$-equivariant truncation of the fermionic Kaluza-Klein tower while maintaining all quantitative features of the continuous reduction, by using fuzzy spinor fields and a universal Dirac operator. Although the classes of models we present here are far from being phenomenologically viable ones, they provide a striking illustration of the utility of equivariant dimensional reduction and how the systematic incorporation of topologically non-trivial gauge fields of the holonomy group can have dramatic implications on the physical particle spectrum of the reduced field theory, including a non-trivial vacuum selection mechanism.

The organisation of this paper is as follows. In $\S 2$ we describe some general aspects of the $\mathrm{SU}(2)$-equivariant dimensional reduction of gauge and fermion fields over $\mathbb{C} P^{1}$. In $\S 3$ we derive the corresponding reduction of the pure massless Yang-Mills-Dirac action functional. In $\S 4$ and $\S 5$ we work out large classes of dynamical symmetry breaking patterns, identifying the entire physical particle spectrum in each case. In $\S 6$ we summarize our findings, discuss some of the open problems not addressed in our analysis, and comment on the possibility of obtaining more physically realistic models using higher-dimensional homogeneous spaces $G / H$ as the internal space.

\section{Equivariant dimensional reduction over $\mathbb{C} P^{1}$}

In this section we will describe the dimensional reduction of gauge and fermion fields over the internal coset space $\mathbb{C} P^{1} \cong \mathrm{SU}(2) / \mathrm{U}(1)$ which are invariant under the action of the $\mathrm{SU}(2)$ isometry group of $\mathbb{C} P^{1}$. It is natural to allow for gauge transformations to accompany the spacetime $\mathrm{SU}(2)$ action $[1,2]$. An elegant and systematic way to implement such a reduction is via a bundle theoretic approach. For more details, see [8, 12].

\subsection{SU(2)-equivariant bundles}

By the inverse relations of induction and restriction [10], there is a one-to-one correspondence between $\mathrm{SU}(2)$-equivariant complex vector bundles $\mathcal{E} \rightarrow \mathcal{M}:=M \times \mathbb{C} P^{1}$ and $\mathrm{U}(1)$-equivariant complex vector bundles $E \rightarrow M$, where $\mathrm{SU}(2)$ acts on the space $\mathcal{M}$ via the trivial action on the manifold $M$ and by the standard (left) transitive action on the projective line $\mathbb{C} P^{1} \cong \mathrm{SU}(2) / \mathrm{U}(1)$. The $\mathrm{U}(1)$ subgroup of $\mathrm{SU}(2)$ also acts trivially on $M$. Assume that the structure group of the principal bundle associated to $\mathcal{E}$ is $\mathrm{U}(k)$. Imposing the condition of $\mathrm{SU}(2)$-equivariance then means that we should look for representations of the isometry group $\mathrm{SU}(2)$ of $\mathbb{C} P^{1}$ inside the $\mathrm{U}(k)$ structure group, i.e. for conjugacy classes of homomorphisms $\rho: \mathrm{SU}(2) \rightarrow \mathrm{U}(k)$. The dimensional reduction is thus given by $k$-dimensional unitary representations of $\mathrm{SU}(2)$. Up to isomorphism, for each positive integer $r$ there is a unique irreducible $\mathrm{SU}(2)$-module $\underline{V}_{r} \cong \mathbb{C}^{r}$ of dimension $r$. Therefore, for each positive integer $m$, the module

$$
\underline{\mathcal{V}}=\bigoplus_{i=0}^{m} \underline{V}_{k_{i}} \quad \text { with } \quad \sum_{i=0}^{m} k_{i}=k
$$

gives a representation $\rho$ of $\mathrm{SU}(2)$ inside $\mathrm{U}(k)$. The original generic $\mathrm{U}(k)$ gauge symmetry then restricts to the centralizer subgroup of the image $\rho(\mathrm{SU}(2))$ in $\mathrm{U}(k)$,

$$
\mathrm{U}(k) \longrightarrow \prod_{i=0}^{m} \mathrm{U}\left(k_{i}\right)
$$


which will be the low-energy gauge group of the dimensionally reduced field theory on $M$. For structure groups $G$ other than $\mathrm{U}(k)$, the homomorphisms $\rho: \mathrm{SU}(2) \rightarrow G$, and hence the analogs of the restriction patterns (2.2), can be deduced from the Dynkin diagram of $G$.

The Lie algebra of $\mathrm{SU}(2)$ is generated by the three Pauli matrices

$$
\sigma_{3}=\left(\begin{array}{cc}
1 & 0 \\
0 & -1
\end{array}\right), \quad \sigma_{+}=\left(\begin{array}{ll}
0 & 1 \\
0 & 0
\end{array}\right) \quad \text { and } \quad \sigma_{-}=\left(\begin{array}{ll}
0 & 0 \\
1 & 0
\end{array}\right)
$$

with the commutation relations

$$
\left[\sigma_{3}, \sigma_{ \pm}\right]= \pm 2 \sigma_{ \pm} \quad \text { and } \quad\left[\sigma_{+}, \sigma_{-}\right]=\sigma_{3}
$$

The Lie algebra of the $\mathrm{U}(1)$ subgroup of $\mathrm{SU}(2)$ is generated in this basis by $\sigma_{3}$. For each $p \in \mathbb{Z}$ there is a unique irreducible representation $\underline{S}_{p} \cong \mathbb{C}$ of $\mathrm{U}(1)$ given by $\zeta \cdot v=\zeta^{p} v$ for $\zeta \in S^{1}$ and $v \in \underline{S}_{p}$. Since the manifold $M$ carries the trivial action of the group U(1), any U(1)-equivariant bundle $E \rightarrow M$ admits a finite Whitney sum decomposition into isotopical components as [16] $E=\bigoplus_{p} E_{(p)} \otimes \underline{S}_{p}$, where the sum runs over the set of eigenvalues for the U(1)-action on $E$ and $E_{(p)} \rightarrow M$ are bundles with the trivial $\mathrm{U}(1)$-action.

The corresponding $\mathrm{SU}(2)$-equivariant bundle $\mathcal{E} \rightarrow M \times \mathbb{C} P^{1}$ is obtained by induction as

$$
\mathcal{E}=\mathrm{SU}(2) \times_{\mathrm{U}(1)} E
$$

where the $\mathrm{U}(1)$-action on $\mathrm{SU}(2) \times E$ is given by $h \cdot(g, e)=\left(g h^{-1}, h \cdot e\right)$ for $h \in \mathrm{U}(1), g \in \mathrm{SU}(2)$ and $e \in E$. The $\sigma_{3}$-action on $\mathcal{E}$ is described by the isotopical decomposition of $E$ above. The rest of the $\mathrm{SU}(2)$ action, i.e. the actions of $\sigma_{+}$and $\sigma_{-}=\sigma_{+}{ }^{\dagger}$, follows from the commutation relations (2.4), which shows that the action of the generator $\sigma_{+}$on $E_{(p)} \otimes \underline{S}_{p}$ corresponds to bundle morphisms $E_{(p)} \rightarrow E_{(p+2)}$, along with the trivial $\sigma_{+}$-actions on the irreducible U(1)-modules $\underline{S}_{p}$. Introduce the standard Dirac $p$-monopole line bundle

$$
\mathcal{L}^{p}:=\mathrm{SU}(2) \times_{\mathrm{U}(1)} \underline{S}_{p}
$$

over the homogeneous space $\mathbb{C} P^{1}$, with $\mathcal{L}^{p}=\mathcal{L}^{\otimes p}$ for $p \geq 0$ and $\mathcal{L}^{p}=\left(\mathcal{L}^{\vee}\right)^{\otimes(-p)}$ for $p<0$ where $\mathcal{L}=\mathcal{L}^{1}$. Then, for the induced complex vector bundle (2.5) over $M \times \mathbb{C} P^{1}$ of rank $k$, the $\sigma_{3}$-action is given by the $\mathrm{U}(1)$-equivariant decomposition

$$
\mathcal{E}=\bigoplus_{i=0}^{m} \mathcal{E}_{i} \quad \text { with } \quad \mathcal{E}_{i}=E_{i} \otimes \mathcal{L}^{p_{i}} \quad \text { and } \quad p_{i}=m-2 i
$$

where $E_{i} \rightarrow M$ are complex vector bundles of rank $k_{i}$ with typical fibre the module $\underline{V}_{k_{i}}$ in (2.1), and $\mathcal{E}_{i} \rightarrow M \times \mathbb{C} P^{1}$ is the bundle with fibres

$$
\left(\mathcal{E}_{i}\right)_{(x, \xi)}=\left(E_{i}\right)_{x} \otimes\left(\mathcal{L}^{p_{i}}\right)_{\xi}
$$

for $x \in M$ and $\xi \in \mathbb{C} P^{1}$. On the other hand, the $\sigma_{+}$-action is determined by a chain

$$
0 \longrightarrow \mathcal{E}_{m} \stackrel{\Phi_{m}}{\longrightarrow} \mathcal{E}_{m-1} \stackrel{\Phi_{m-1}}{\longrightarrow} \cdots \stackrel{\Phi_{2}}{\longrightarrow} \mathcal{E}_{1} \stackrel{\Phi_{1}}{\longrightarrow} \mathcal{E}_{0} \longrightarrow 0
$$

of bundle morphisms between consecutive $\mathcal{E}_{i}$ 's. After fixing hermitean metrics on the complex vector bundles $\mathcal{E}_{i} \rightarrow \mathcal{M}$, the $\sigma_{-}$-action is described by reversing the arrows in (2.9) and using the adjoint bundle morphisms $\Phi_{i}^{\dagger}$. 
This decomposition can be understood as follows. Given any finite-dimensional representation $\underline{V}$ of $\mathrm{U}(1)$, the corresponding induced, homogeneous hermitean vector bundle over the coset space $\mathbb{C} P^{1} \cong \mathrm{SU}(2) / \mathrm{U}(1)$ is given by the fibred product

$$
\mathcal{V}=\mathrm{SU}(2) \times_{\mathrm{U}(1)} \underline{V} .
$$

Every $\mathrm{SU}(2)$-equivariant bundle of finite rank over $\mathbb{C} P^{1}$, with respect to the standard transitive action of $\mathrm{SU}(2)$ on the homogeneous space, is of the form (2.10). If $\underline{V}$ is irreducible, then $\mathrm{U}(1)$ is the structure group of the associated principal bundle. We consider those representations $\underline{V}$ which descend from some irreducible representation of $\mathrm{SU}(2)$ by restriction to the $\mathrm{U}(1)$ subgroup. Then the bundle decomposition (2.7) is associated with the restriction of the irreducible $\mathrm{SU}(2)$ representation of dimension $r=m+1$.

\subsection{Invariant gauge fields}

Let $M$ be a manifold of real dimension $d$ with local real coordinates $x=\left(x^{\mu}\right) \in \mathbb{R}^{d}$, where the indices $\mu, \nu, \ldots$ run through $1, \ldots, d$. The projective line $\mathbb{C} P^{1}$ is a complex manifold with local complex coordinate $y \in \mathbb{C}$ and its conjugate $\bar{y}$. The metric

$$
\mathrm{d} s^{2}=\mathcal{G}_{A B} \mathrm{~d} x^{A} \otimes \mathrm{d} x^{B}
$$

on $\mathcal{M}=M \times \mathbb{C} P^{1}$ will be taken to be the direct product of a chosen riemannian metric on $M$ and the standard $\mathrm{SO}(3)$-symmetric metric on $\mathbb{C} P^{1} \cong S^{2}$, where the indices $A, B, \ldots$ run over $1, \ldots, d+2$. In the coordinates above it takes the form

$$
\mathrm{d} s^{2}=G_{\mu \nu} \mathrm{d} x^{\mu} \otimes \mathrm{d} x^{\nu}+\frac{4 R^{2}}{(1+y \bar{y})^{2}} \mathrm{~d} y \otimes \mathrm{d} \bar{y}
$$

where $R$ is the radius of the sphere $S^{2}$. We use conventions in which the coordinates $x$ and $y$ are dimensionless, while the line element (2.12) has mass dimension -2 . More generally, one may consider warped compactifications of $\mathcal{M}$ with the same topology, but this doesn't seem to add any new qualitative features to our ensuing results.

Let $\mathcal{A}$ be a connection on the hermitean vector bundle $\mathcal{E} \rightarrow M \times \mathbb{C} P^{1}$ having the form given by $\mathcal{A}=\mathcal{A}_{A} \mathrm{~d} x^{A}$ in local coordinates $\left(x^{A}\right)$ and taking values in the Lie algebra $\mathrm{u}(k)$. We will now describe the $\mathrm{SU}(2)$-equivariant reduction of $\mathcal{A}$ on $M \times \mathbb{C} P^{1}$. The spherical dependences are in this case completely determined by the rank $k$ of the bundle $\mathcal{E}$ and the unique (up to gauge transformations) $\mathrm{SU}(2)$-invariant connections $a_{p}$ on the monopole line bundles (2.6) having, in local complex coordinates on $\mathbb{C} P^{1}$, the forms

$$
a_{p}=\frac{p}{2(1+y \bar{y})}(\bar{y} \mathrm{~d} y-y \mathrm{~d} \bar{y}) .
$$

The curvatures of these connections are

$$
f_{p}=\mathrm{d} a_{p}=-\frac{p}{(1+y \bar{y})^{2}} \mathrm{~d} y \wedge \mathrm{d} \bar{y}
$$

and their topological charges are given by the degrees of the complex line bundles $\mathcal{L}^{p} \rightarrow \mathbb{C} P^{1}$ as

$$
\operatorname{deg} \mathcal{L}^{p}=\frac{\mathrm{i}}{2 \pi} \int_{\mathbb{C} P^{1}} f_{p}=p
$$


Related to the monopole fields are the unique, covariantly constant $\mathrm{SU}(2)$-invariant forms of types $(1,0)$ and $(0,1)$ on $\mathbb{C} P^{1}$ given respectively by

$$
\beta=\frac{2 \mathrm{~d} y}{1+y \bar{y}} \quad \text { and } \quad \bar{\beta}=\frac{2 \mathrm{~d} \bar{y}}{1+y \bar{y}} .
$$

They respectively form a basis of sections of the canonical line bundles $K=\mathcal{L}^{2}$ and $K^{-1}=\mathcal{L}^{-2}$, which are the summands of the complexified cotangent bundle $T^{*} \mathbb{C} P^{1} \otimes \mathbb{C}=K \oplus K^{-1}$ over $\mathbb{C} P^{1}$. The $\mathrm{SU}(2)$-invariant Kähler (1,1)-form on $\mathbb{C} P^{1}$ is $\frac{\mathrm{i}}{2} R^{2} \beta \wedge \bar{\beta}$.

With respect to the isotopical decomposition (2.7), the twisted $\mathrm{u}(k)$-valued gauge potential $\mathcal{A}$ splits into $k_{i} \times k_{j}$ blocks $\mathcal{A}=\left(\mathcal{A}^{i j}\right)$ with $\mathcal{A}^{i j} \in \operatorname{Hom}\left(\underline{V}_{k_{j}}, \underline{V}_{k_{i}}\right)$, which we write as

$$
\mathcal{A}=\boldsymbol{A}^{(m)}(x) \otimes 1+\mathbf{1}_{k} \otimes \boldsymbol{a}^{(m)}(y)+\boldsymbol{\phi}_{(m)}(x) \otimes \bar{\beta}(y)-\left(\phi_{(m)}(x)\right)^{\dagger} \otimes \beta(y)
$$

where

$$
\phi_{(m)}:=\left(\begin{array}{ccccc}
0 & \phi_{1} & 0 & \ldots & 0 \\
0 & 0 & \phi_{2} & \ldots & 0 \\
\vdots & \vdots & \ddots & \ddots & \vdots \\
0 & 0 & 0 & \ldots & \phi_{m} \\
0 & 0 & 0 & \ldots & 0
\end{array}\right)
$$

while

$$
\boldsymbol{A}^{(m)}:=\sum_{i=0}^{m} A^{i} \otimes \Pi_{i} \quad \text { and } \quad \boldsymbol{a}^{(m)}:=\sum_{i=0}^{m} a_{p_{i}} \otimes \Pi_{i}
$$

with $\Pi_{i}: \mathcal{E} \rightarrow \mathcal{E}_{i}$ the canonical orthogonal projections of rank one onto the sub-bundles $\mathcal{E}_{i}$, obeying $\Pi_{i} \Pi_{j}=\delta_{i j} \Pi_{i}$. The bundle morphisms $\Phi_{i+1}:=\mathcal{A}^{i i+1}=\phi_{i+1}(x) \otimes \bar{\beta}(y) \in \operatorname{Hom}\left(\mathcal{E}_{i+1}, \mathcal{E}_{i}\right)$ obey $\Phi_{m+1}=0=\Phi_{0}$. The gauge potentials $A^{i} \in \mathrm{u}\left(k_{i}\right)$ are connections on the hermitean vector bundles $E_{i} \rightarrow M$. The bifundamental scalar fields $\phi_{i+1} \in \operatorname{Hom}\left(E_{i+1}, E_{i}\right)$ can be identified with sections of the bundles $E_{i} \otimes E_{i+1}^{\vee}$ and transform in the representations $\underline{V}_{k_{i}} \otimes \underline{V}_{k_{i+1}}^{\vee}$ of the subgroups $\mathrm{U}\left(k_{i}\right) \times \mathrm{U}\left(k_{i+1}\right)$ of the original $\mathrm{U}(k)$ gauge group. The gauge potential $\mathcal{A}$ given by (2.17) is antihermitean and $\mathrm{SO}(3)$-invariant. All fields $\left(A^{i}, \phi_{i+1}\right)$ are dimensionless and depend only on the coordinates $x \in M$. Every SU(2)-invariant unitary connection $\mathcal{A}$ on $M \times \mathbb{C} P^{1}$ is of the form given in $(2.17)$ (up to gauge transformations) $[12,10]$.

The curvature two-form $\mathcal{F}=\mathrm{d} \mathcal{A}+\mathcal{A} \wedge \mathcal{A}$ of the connection $\mathcal{A}$ has components which are given by $\mathcal{F}_{A B}=\partial_{A} \mathcal{A}_{B}-\partial_{B} \mathcal{A}_{A}+\left[\mathcal{A}_{A}, \mathcal{A}_{B}\right]$ in local coordinates $\left(x^{A}\right)$, where $\partial_{A}:=\partial / \partial x^{A}$. It also takes values in the Lie algebra $\mathrm{u}(k)$, and in local coordinates on $M \times \mathbb{C} P^{1}$ it can be written as

$$
\mathcal{F}=\frac{1}{2} \mathcal{F}_{\mu \nu} \mathrm{d} x^{\mu} \wedge \mathrm{d} x^{\nu}+\mathcal{F}_{\mu y} \mathrm{~d} x^{\mu} \wedge \mathrm{d} y+\mathcal{F}_{\mu \bar{y}} \mathrm{~d} x^{\mu} \wedge \mathrm{d} \bar{y}+\mathcal{F}_{y \bar{y}} \mathrm{~d} y \wedge \mathrm{d} \bar{y} .
$$

The calculation of the curvature (2.20) for $\mathcal{A}$ of the form (2.17) yields

$$
\mathcal{F}=\left(\mathcal{F}^{i j}\right) \quad \text { with } \quad \mathcal{F}^{i j}=\mathrm{d} \mathcal{A}^{i j}+\sum_{l=0}^{m} \mathcal{A}^{i l} \wedge \mathcal{A}^{l j},
$$

giving

$$
\mathcal{F}=\boldsymbol{F}^{(m)}+\boldsymbol{f}^{(m)}+\left[\boldsymbol{\phi}_{(m)}, \boldsymbol{\phi}_{(m)}^{\dagger}\right] \beta \wedge \bar{\beta}+D \boldsymbol{\phi}_{(m)} \wedge \bar{\beta}-\left(D \boldsymbol{\phi}_{(m)}\right)^{\dagger} \wedge \beta
$$

where

$$
\boldsymbol{F}^{(m)}:=\mathrm{d} \boldsymbol{A}^{(m)}+\boldsymbol{A}^{(m)} \wedge \boldsymbol{A}^{(m)} \quad \text { and } \quad D \boldsymbol{\phi}_{(m)}:=\mathrm{d} \boldsymbol{\phi}_{(m)}+\left[\boldsymbol{A}^{(m)}, \boldsymbol{\phi}_{(m)}\right],
$$


while $\boldsymbol{f}^{(m)}=\mathrm{d} \boldsymbol{a}^{(m)}=\sum_{i} f_{p_{i}} \otimes \Pi_{i}$ are the contributions from the monopole fields. We have suppressed the tensor product structure pertaining to $M \times \mathbb{C} P^{1}$. From (2.22) we find the nonvanishing field strength components

$$
\begin{aligned}
\mathcal{F}_{\mu \nu}^{i i} & =F_{\mu \nu}^{i} \\
\mathcal{F}_{\mu \bar{y}}^{i i+1} & =\frac{2}{1+y \bar{y}} D_{\mu} \phi_{i+1}=-\left(\mathcal{F}_{\mu y}^{i+1 i}\right)^{\dagger}, \\
\mathcal{F}_{y \bar{y}}^{i i} & =-\frac{1}{(1+y \bar{y})^{2}}\left(p_{i}+4 \phi_{i}^{\dagger} \phi_{i}-4 \phi_{i+1} \phi_{i+1}^{\dagger}\right),
\end{aligned}
$$

where $F^{i}=\mathrm{d} A^{i}+A^{i} \wedge A^{i}=\frac{1}{2} F_{\mu \nu}^{i} \mathrm{~d} x^{\mu} \wedge \mathrm{d} x^{\nu}$ are the curvatures of the bundles $E_{i} \rightarrow M$, and

$$
D \phi_{i+1}=\mathrm{d} \phi_{i+1}+A^{i} \phi_{i+1}-\phi_{i+1} A^{i+1}
$$

are bifundamental covariant derivatives.

The gauge field (2.22) can be formally identified with the lowest $\mathrm{SU}(2)$-singlet mode in a harmonic expansion of forms on the internal space $\mathbb{C} P^{1}$. Since the monopole fields are given by $f_{p_{i}}=-\frac{p_{i}}{4} \beta \wedge \bar{\beta}$, it can be uniquely characterized by the requirement that it lives in the kernel of the covariant derivative operator on $\mathbb{C} P^{1}$ in the monopole background, owing to the relations

$$
\mathrm{d} \bar{\beta}-a_{-2} \wedge \bar{\beta}=0=\mathrm{d} \beta-a_{2} \wedge \beta
$$

Equivalently, it is a zero mode of the covariant Laplace operator acting on forms on $\mathbb{C} P^{1}$. As usual in Kaluza-Klein reductions, there is an infinite tower of massive harmonic modes on $M$ which can also be considered. Their contributions will not be analysed in this paper.

\subsection{Symmetric spinor fields}

Let $M$ be a spin manifold. When $d=\operatorname{dim}_{\mathbb{R}}(M)$ is even, the generators of the Clifford algebra $\mathrm{C} \ell\left(M \times \mathbb{C} P^{1}\right)$ obey

$$
\Gamma^{A} \Gamma^{B}+\Gamma^{B} \Gamma^{A}=-2 \mathcal{G}^{A B} \mathbf{1}_{2^{d / 2+1}} \quad \text { with } \quad A, B=1, \ldots, d+2 .
$$

The gamma-matrices in (2.29) may be decomposed as

$$
\left\{\Gamma^{A}\right\}=\left\{\Gamma^{\mu}, \Gamma^{y}, \Gamma^{\bar{y}}\right\} \quad \text { with } \quad \Gamma^{\mu}=\gamma^{\mu} \otimes \mathbf{1}_{2}, \quad \Gamma^{y}=\gamma \otimes \gamma^{y} \quad \text { and } \quad \Gamma^{\bar{y}}=\gamma \otimes \gamma^{\bar{y}},
$$

where the $2^{d / 2} \times 2^{d / 2}$ matrices $\gamma^{\mu}=-\left(\gamma^{\mu}\right)^{\dagger}$ act locally on the spinor module $\underline{\Delta}(M)$ over the Clifford algebra bundle $\mathrm{C} \ell(M) \rightarrow M$,

$$
\gamma^{\mu} \gamma^{\nu}+\gamma^{\nu} \gamma^{\mu}=-2 G^{\mu \nu} \mathbf{1}_{2^{d / 2}} \quad \text { with } \quad \mu, \nu=1, \ldots, d,
$$

while

$$
\gamma=\frac{\mathrm{i}^{d / 2} \sqrt{G}}{d !} \epsilon_{\mu_{1} \cdots \mu_{d}} \gamma^{\mu_{1}} \cdots \gamma^{\mu_{d}} \quad \text { with } \quad(\gamma)^{2}=\mathbf{1}_{2^{d / 2}} \quad \text { and } \quad \gamma \gamma^{\mu}=-\gamma^{\mu} \gamma
$$

is the corresponding chirality operator. Here $\epsilon_{\mu_{1} \ldots \mu_{d}}$ is the Levi-Civita symbol with $\epsilon_{12 \cdots d}=+1$. The action of the Clifford algebra $\mathrm{C} \ell\left(\mathbb{C} P^{1}\right)$ on the spinor module $\underline{\Delta}\left(\mathbb{C} P^{1}\right)$ is generated by

$$
\gamma^{y}=-\frac{1}{2 R}(1+y \bar{y}) \sigma_{+} \quad \text { and } \quad \gamma^{\bar{y}}=\frac{1}{2 R}(1+y \bar{y}) \sigma_{-} .
$$


The treatment for $d$ odd is similar.

The $\mathcal{E}$-twisted Dirac operator on $\mathcal{M}=M \times \mathbb{C} P^{1}$ corresponding to the equivariant gauge potential $\mathcal{A}$ in $(2.17)$ is given by

$$
\not \mathcal{D}:=\Gamma^{A} \mathcal{D}_{A}=\gamma^{\mu} D_{\mu} \otimes \mathbf{1}_{2}+\left(\phi_{(m)}\right) \gamma \otimes \gamma^{\bar{y}} \beta_{\bar{y}}-\left(\phi_{(m)}\right)^{\dagger} \gamma \otimes \gamma^{y} \beta_{y}+\gamma \otimes \not D_{\mathbb{C} P^{1}}
$$

where

$$
\not D_{\mathbb{C} P^{1}}:=\gamma^{y} D_{y}+\gamma^{\bar{y}} D_{\bar{y}}=\gamma^{y}\left(\partial_{y}+\omega_{y}+\left(\boldsymbol{a}^{(m)}\right)_{y}\right)+\gamma^{\bar{y}}\left(\partial_{\bar{y}}+\omega_{\bar{y}}+\left(\boldsymbol{a}^{(m)}\right)_{\bar{y}}\right)
$$

and $\omega_{y}, \omega_{\bar{y}}$ are the components of the Levi-Civita spin connection on the tangent bundle of $\mathbb{C} P^{1}$. The $E$-twisted Dirac operator $\not D:=\gamma^{\mu} D_{\mu}$ on $M$ is defined as

$$
\not D=\gamma^{\mu}\left(\partial_{\mu}+\theta_{\mu}+\left(\boldsymbol{A}^{(m)}\right)_{\mu}\right) \quad \text { with } \quad \theta_{\mu}=\frac{1}{2} \theta_{\mu \nu \lambda} \Sigma^{\nu \lambda}
$$

where $\theta=\theta_{\mu} \mathrm{d} x^{\mu}$ is the spin connection on the tangent bundle of the manifold $M$ and $\Sigma^{\nu \lambda}$ are the generators of $\operatorname{Spin}(d)$. The operator (2.34) acts on spinors $\Psi$ which are $\mathrm{L}^{2}$-sections of the bundle

$$
\Psi=\left(\begin{array}{l}
\Psi^{+} \\
\Psi^{-}
\end{array}\right) \in \bigoplus_{i=0}^{m}\left(E_{i} \otimes \Delta(M)\right) \otimes\left(\begin{array}{l}
\mathcal{L}^{p_{i}+1} \\
\mathcal{L}^{p_{i}-1}
\end{array}\right)
$$

over $M \times \mathbb{C} P^{1}$, where $\mathcal{L}^{p_{i}+1} \oplus \mathcal{L}^{p_{i}-1}$ are the twisted spinor bundles of rank two over the sphere $\mathbb{C} P^{1}$ and $\Psi^{ \pm}$are $2^{d / 2+1}$ component spinors satisfying $\left(\mathbf{1}_{2^{d / 2}} \otimes \sigma_{3}\right) \Psi^{ \pm}= \pm \Psi^{ \pm}$.

The equivariant dimensional reduction of massless Dirac spinors on $M \times \mathbb{C} P^{1}$ is defined with respect to symmetric fermions on $M$. Similarly to the scalar fields $\phi_{i+1}(x)$ in $(2.17)$, they act as intertwining operators connecting induced representations of $\mathrm{U}(1)$ in the $\mathrm{U}(k)$ gauge group, and also in the spinor module $\underline{\Delta}(M)$ which admits the isotopical decomposition

$$
\underline{\Delta}(M)=\bigoplus_{i=0}^{m} \Delta_{i} \otimes \underline{S}_{p_{i}} \quad \text { with } \quad \Delta_{i}=\operatorname{Hom}_{\mathrm{U}(1)}\left(\underline{S}_{p_{i}}, \underline{\Delta}(M)\right)
$$

obtained by restricting $\underline{\Delta}(M)$ to representations of $\mathrm{U}(1) \subset \operatorname{Spin}(d) \subset \mathrm{C} \ell(M)$. The $\Delta_{i}$ 's in (2.38) are the corresponding multiplicity spaces, and using Frobenius reciprocity they may be identified as

$$
\Delta_{i}=\operatorname{Hom}_{\mathrm{SU}(2)}\left(\underline{\Delta}(M), \mathrm{L}^{2}\left(\mathbb{C} P^{1}, \mathcal{L}^{p_{i}}\right)\right)
$$

The isotopical decomposition (2.38) is now realized explicitly by using (2.39) to construct symmetric fermions on $M$ as $\mathrm{SU}(2)$-invariant spinors on $M \times \mathbb{C} P^{1}$. Analogously to the invariant gauge fields, they belong to the kernel of the Dirac operator $(2.35)$ on $\mathbb{C} P^{1}$, and after dimensional reduction will be massless on $M$. One can write

$$
\not D_{\mathbb{C} P^{1}}=\bigoplus_{i=0}^{m} \not D_{p_{i}}=\bigoplus_{i=0}^{m}\left(\begin{array}{cc}
0 & \not D_{p_{i}}^{-} \\
\not D_{p_{i}}^{+} & 0
\end{array}\right)
$$

where

$$
\begin{aligned}
& \not p_{p_{i}}^{+}=\frac{1}{2 R}\left[(1+y \bar{y}) \partial_{\bar{y}}-\frac{1}{2}\left(p_{i}+1\right) y\right] \\
& \not p_{p_{i}}^{-}=-\frac{1}{2 R}\left[(1+y \bar{y}) \partial_{y}+\frac{1}{2}\left(p_{i}-1\right) \bar{y}\right]
\end{aligned}
$$


The operator (2.40) acts on sections of the bundle (2.37) which we write with respect to this decomposition as

$$
\Psi=\bigoplus_{i=0}^{m}\left(\begin{array}{l}
\Psi_{\left(p_{i}\right)}^{+} \\
\Psi_{\left(p_{i}\right)}^{-}
\end{array}\right)
$$

where $\Psi_{\left(p_{i}\right)}^{ \pm}$are $L^{2}$-sections of $\mathcal{L}^{p_{i} \pm 1}$ taking values in $\underline{\Delta}(M) \otimes \underline{V}_{k_{i}}$ with coefficients depending on $x \in M$.

We need to solve the differential equations

$$
\not p_{p_{i}}^{+} \Psi_{\left(p_{i}\right)}^{+}=0 \quad \text { and } \quad \not p_{p_{i}}^{-} \Psi_{\left(p_{i}\right)}^{-}=0
$$

for the spinors $\Psi_{\left(p_{i}\right)}^{+}$and $\Psi_{\left(p_{i}\right)}^{-}$in ker $\not_{p_{i}}^{+}$and ker $\not_{p_{i}}^{-}$. By using the forms of the transition functions for the monopole bundles, one easily sees that the only solutions of these equations which are regular on both the northern and southern hemispheres of $S^{2}$ are of the form

$$
\begin{aligned}
& \Psi_{\left(p_{i}\right)}^{+}=\sum_{\ell=0}^{-p_{i}-1} \psi_{\left(p_{i}\right) \ell}(x) \otimes \chi_{\left(p_{i}\right) \ell}^{+}(y, \bar{y}) \quad \text { and } \quad \Psi_{\left(p_{i}\right)}^{-}=0 \quad \text { for } \quad p_{i}<0, \\
& \Psi_{\left(p_{i}\right)}^{-}=\sum_{\ell=0}^{p_{i}-1} \widetilde{\psi}_{\left(p_{i}\right) \ell}(x) \otimes \chi_{\left(p_{i}\right) \ell}^{-}(y, \bar{y}) \quad \text { and } \quad \Psi_{\left(p_{i}\right)}^{+}=0 \quad \text { for } \quad p_{i}>0,
\end{aligned}
$$

with

$$
\chi_{\left(p_{i}\right) \ell}^{+}(y, \bar{y})=\frac{y^{\ell}}{(1+y \bar{y})^{-\left(p_{i}+1\right) / 2}} \quad \text { and } \quad \chi_{\left(p_{i}\right) \ell}^{-}(y, \bar{y})=\frac{\bar{y}^{\ell}}{(1+y \bar{y})^{\left(p_{i}-1\right) / 2}} .
$$

The components, $\psi_{\left(p_{i}\right) \ell}(x)$ and $\widetilde{\psi}_{\left(p_{i}\right) \ell}(x)$ with $\ell=0,1, \ldots,\left|p_{i}\right|-1$, are Dirac spinors on $M$ which form the irreducible representation $\underline{V}_{\left|p_{i}\right|} \cong \mathbb{C}^{\left|p_{i}\right|}$ of the group $\mathrm{SU}(2)$. This is of course consistent with the fact that the index of the Dirac operator $\mathbb{D}_{p}$ is equal to $-p$.

\subsection{Harmonic spinor fields}

In contrast to the bosonic sector, in the following we will find some noteworthy features of higher Kaluza-Klein modes in the fermionic sector, so we shall describe them as well for completeness. They correspond to eigenspinors with non-zero eigenvalues in the spectrum of the Dirac operator (2.40) on $\mathbb{C} P^{1}$, and are the only surviving fermions in the absence of the monopole background. The twisted spinor bundle given by $\mathcal{L}^{p_{i}+1} \oplus \mathcal{L}^{p_{i}-1}$ admits an infinite-dimensional vector space of symmetric $\mathrm{L}^{2}$-sections comprised of spinor harmonics $\Psi_{j, p_{i}} \in \mathbb{C}^{2}$, with $p_{i}=m-2 i$ [17]. They are eigenspinors of the Dirac operator $\not_{p_{i}}, D_{p_{i}} \Psi_{j, p_{i}}= \pm \frac{1}{R} \lambda_{j, p_{i}} \Psi_{j, p_{i}}$, with eigenvalues

$$
\lambda_{j, p_{i}}=\sqrt{\left(j+\frac{1-p_{i}}{2}\right)\left(j+\frac{1+p_{i}}{2}\right)}
$$

each of multiplicity

$$
d_{j}=2 j+1
$$

where $j$ is integral for odd $p_{i}$ and half-integral for even $p_{i}$ with $j \geq \frac{\left|p_{i}\right|+1}{2}$. After dimensional reduction, this produces an infinite Kaluza-Klein tower of massive Dirac spinors on $M$. We decompose 
the spinors $(2.43)$ in this case as

$$
\begin{aligned}
& \Psi_{\left(p_{i}\right)}^{+}=\sum_{j=\frac{\left|p_{i}\right|+1}{2}}^{\infty} \sum_{\ell=0}^{2 j} \psi_{\left(j, p_{i}\right) \ell}(x) \otimes \chi_{\left(j, p_{i}\right) \ell}^{+}(y, \bar{y}), \\
& \Psi_{\left(p_{i}\right)}^{-}=\sum_{j=\frac{\left|p_{i}\right|+1}{2}}^{\infty} \sum_{\ell=0}^{2 j} \widetilde{\psi}_{\left(j, p_{i}\right) \ell}(x) \otimes \chi_{\left(j, p_{i}\right) \ell}^{-}(y, \bar{y}),
\end{aligned}
$$

where $\chi_{\left(j, p_{i}\right) \ell}^{ \pm}$are the chiral and antichiral spinors which are sections of $\mathcal{L}^{p_{i} \pm 1}$, and form an $\mathrm{L}^{2}$ orthogonal system on $\mathbb{C} P^{1}$ normalized as $\left\|\chi_{\left(j, p_{i}\right) \ell}^{ \pm}\right\|_{\mathrm{L}^{2}}=4 \pi R^{2}$ with

$$
\not p_{p_{i}}^{ \pm} \chi_{\left(j, p_{i}\right) \ell}^{ \pm}=\frac{1}{R} \lambda_{j, p_{i}} \chi_{\left(j, p_{i}\right) \ell}^{\mp}
$$

for each $\ell=0,1, \ldots, 2 j$. The $\left|p_{i}\right|$ zero modes when $\left|p_{i}\right| \geq 1$ are recovered for $j=\frac{1}{2}\left(\left|p_{i}\right|-1\right)$.

In contrast to the zero mode sector, this sector of the dimensionally reduced field theory contains an infinite number of modes on $M$, indicated by the infinite range of the angular momenta $j$. In this case a natural SU(2)-invariant way of reducing to a finite number of fermionic field degrees of freedom is to use a fuzzy sphere $\mathbb{C} P_{F}^{1}[18]$, truncated at some finite level $j=j_{\text {max }}$, as the internal space. Dimensional reduction on the fuzzy sphere was considered in [19], although only for the case $m=0$ with no background monopole fields. Kaluza-Klein compactifications on $\mathbb{C} P_{F}^{1}$ including non-trivial internal magnetic flux are studied in [20], although in a different context than ours and in somewhat less generality.

The Dirac equation on the fuzzy sphere, and more generally on fuzzy $\mathbb{C} P^{N}$, has been analysed in [21] and [22] respectively. The spectrum of the (universal) fuzzy Dirac operator including monopole backgrounds for a given maximal angular momentum $j_{\max }$ consists again of the eigenvalues (2.48) as in the continuous case, except that now $j \leq j_{\max }$. The corresponding eigenspinors can be constructed as finite-dimensional matrices. With $L=j_{\max }+\frac{1}{2}$, positive chirality spinors $\hat{\chi}_{(j, p) \ell}^{+}$are complex matrices of dimension given by $\left(L-\frac{p}{2}\right) \times\left(L+1+\frac{p}{2}\right)$, while negative chirality spinors $\hat{\chi}_{\left(j, p^{\prime}\right) \ell}^{-}$are matrices of dimension $\left(L+1-\frac{p^{\prime}}{2}\right) \times\left(L+\frac{p^{\prime}}{2}\right)$. By truncating at $j_{\max }=\frac{\left|p_{i}\right|-1}{2}$, all spinor fields $\psi_{\left(j, p_{i}\right) \ell}$ and $\widetilde{\psi}_{\left(j, p_{i}\right) \ell}$ vanish identically, and only the finitely many flavours of the zero-mode symmetric spinors $\psi_{\left(p_{i}\right) \ell}$ and $\widetilde{\psi}_{\left(p_{i}\right) \ell}$ of $\S 2.3$ survive the dimensional reduction.

\section{$3 \quad$ Equivariant gauge theory of Kaluza-Klein modes}

In this section we will work out the equivariant dimensional reduction of the pure massless YangMills-Dirac action on $M \times \mathbb{C} P^{1}$. We will find that the role of the monopole fields on $\mathbb{C} P^{1}$ is to induce a Higgs potential with dynamical symmetry breaking, as well as couplings to massless spinors with Yukawa interactions from the zero modes of the Dirac operator $D_{\mathbb{C} P^{1}}$. The mass scale of the broken symmetry phase on $M$ is determined by the size $R$ of the internal coset space. An induced Yukawa sector of the low-energy effective field theory then emerges with the standard form of spontaneous symmetry breaking, containing both massless and massive fermions together with Yukawa interactions with the physical Higgs fields. In particular, we will unveil the possibility of obtaining exactly massless chiral fermions on $M$ with Yukawa interactions, which can be interpreted as multiplets of left-handed quarks. Our approach thus avoids the extra requirements necessary for obtaining chiral fermions in the more conventional coset space dimensional reduction schemes [2]. 


\subsection{Dimensional reduction of the Yang-Mills action}

For the usual Yang-Mills lagrangian

$$
L_{\mathrm{YM}}=-\frac{1}{4 \tilde{g}^{2}} \sqrt{|\mathcal{G}|} \operatorname{tr}_{k \times k} \mathcal{F}_{A B} \mathcal{F}^{A B}
$$

on $\mathcal{M}=M \times \mathbb{C} P^{1}$, one has

$$
\begin{gathered}
L_{\mathrm{YM}}=-\frac{1}{4 \tilde{g}^{2}} \sqrt{|\mathcal{G}|} \operatorname{tr}_{k \times k}\left[\mathcal{F}_{\mu \nu} \mathcal{F}^{\mu \nu}+\frac{(1+y \bar{y})^{2}}{2 R^{2}} G^{\mu \nu}\left(\mathcal{F}_{\mu y} \mathcal{F}_{\nu \bar{y}}+\mathcal{F}_{\mu \bar{y}} \mathcal{F}_{\nu y}\right)\right. \\
\left.+\frac{1}{8}\left(\frac{(1+y \bar{y})^{2}}{R^{2}} \mathcal{F}_{y \bar{y}}\right)^{2}\right] .
\end{gathered}
$$

The $(d+2)$-dimensional $\mathrm{U}(k)$ Yang-Mills coupling constant $\tilde{g}$ has the standard mass dimension $1-\frac{d}{2}$ in order to make (3.2) dimensionless. The dimensional reduction of the corresponding Yang-Mills action can be obtained by substituting (2.24)-(2.26) into (3.2) and performing the integral over $\mathbb{C} P^{1}$ to arrive at the action

$$
\begin{aligned}
S_{\mathrm{YM}}:= & \int_{M \times \mathbb{C} P^{1}} \mathrm{~d}^{d+2} x L_{\mathrm{YM}} \\
= & \frac{\pi R^{2}}{\tilde{g}^{2}} \int_{M} \mathrm{~d}^{d} x \sqrt{|G|} \sum_{i=0}^{m} \operatorname{tr}_{k_{i} \times k_{i}}\left[\left(F_{\mu \nu}^{i}\right)^{\dagger}\left(F^{i \mu \nu}\right)+\frac{2}{R^{2}}\left(D_{\mu} \phi_{i+1}\right)\left(D^{\mu} \phi_{i+1}\right)^{\dagger}\right. \\
& \left.+\frac{2}{R^{2}}\left(D_{\mu} \phi_{i}\right)^{\dagger}\left(D^{\mu} \phi_{i}\right)+\frac{1}{8 R^{4}}\left(p_{i}+4 \phi_{i}^{\dagger} \phi_{i}-4 \phi_{i+1} \phi_{i+1}^{\dagger}\right)^{2}\right] .
\end{aligned}
$$

This result holds irrespectively of the signature of the chosen metric on the manifold $M$.

The action (3.3) defines a non-abelian Higgs model describing $m$ interacting complex "rectangular" scalar fields coupled to $m+1$ non-abelian gauge fields. From (2.27) it follows that the $\mathrm{U}(1)$ factor in $\mathrm{U}(k) \approx \mathrm{SU}(k) \times \mathrm{U}(1)$ does not enter the bicovariant derivatives of $\phi_{i+1}$, since an overall $\mathrm{U}(1)$ factor cancels between the $A^{i}$ and the $A^{i+1}$ terms. For the purposes of the ensuing analysis in this subsection we can therefore focus on gauge group $\mathrm{SU}(k)$, though the overall $\mathrm{U}(1)$ subgroup would in general couple to fermions, in the fundamental representation of $\mathrm{SU}(k)$ for example. The decomposition (2.2) of the gauge group arising from the regular embedding of $\mathrm{SU}(2)$ is then modified to

$$
\mathrm{SU}(k) \longrightarrow \mathrm{U}(1)^{m} \times \prod_{i=0}^{m} \mathrm{SU}\left(k_{i}\right) \quad \text { with } \quad \sum_{i=0}^{m} k_{i}=k
$$

The gauge coupling in $d$ dimensions should have mass dimension $2-\frac{d}{2}$, so we define $g^{2}=\tilde{g}^{2} / 4 \pi R^{2}$ as the $d$-dimensional gauge coupling constant. We then rescale $\phi_{i} \rightarrow g R \phi_{i}$ and $A^{i} \rightarrow g A^{i}$ so that the scalar fields and the gauge fields have the correct canonical dimensions for a $d$-dimensional field theory (with dimensionless coordinates).

The action (3.3) can be succinctly rewritten as a matrix model by using the operators (2.18), (2.19) and (2.23) (with the rescalings $\boldsymbol{\phi}_{(m)} \rightarrow g R \boldsymbol{\phi}_{(m)}$ and $\boldsymbol{A}^{(m)} \rightarrow g \boldsymbol{A}^{(m)}$ ), together with

$$
\boldsymbol{\Sigma}_{(m)}:=\sum_{i=0}^{m} p_{i} \Pi_{i}
$$

with respect to the decomposition (2.7). One then has

$$
S_{\mathrm{YM}}=\int_{M} \mathrm{~d}^{d} x \sqrt{|G|}\left[\operatorname{tr}_{k \times k}\left(\frac{1}{4}\left(\boldsymbol{F}^{(m)}\right)_{\mu \nu}^{\dagger}\left(\boldsymbol{F}^{(m)}\right)^{\mu \nu}+\left(D_{\mu} \boldsymbol{\phi}_{(m)}\right)^{\dagger}\left(D^{\mu} \boldsymbol{\phi}_{(m)}\right)\right)+V\left(\boldsymbol{\phi}_{(m)}\right)\right] \text {, }
$$


where the Higgs potential is given by

$$
V\left(\phi_{(m)}\right)=\frac{g^{2}}{2} \operatorname{tr}_{k \times k}\left(\frac{1}{4 g^{2} R^{2}} \boldsymbol{\Sigma}_{(m)}-\left[\phi_{(m)}, \phi_{(m)}^{\dagger}\right]\right)^{2} .
$$

The Higgs potential (3.7) generically leads to dynamical symmetry breaking, as a direct consequence of the non-trivial monopole background on $\mathbb{C} P^{1}$. Its critical points are described by the matrix equations

$$
\phi_{(m)}-2 g^{2} R^{2}\left[\left[\phi_{(m)}, \phi_{(m)}^{\dagger}\right], \phi_{(m)}\right]=0,
$$

where we have used $\left[\boldsymbol{\Sigma}_{(m)}, \phi_{(m)}\right]=2 \phi_{(m)}$. When they exist, solutions of the equation

$$
\left[\phi_{(m)}, \phi_{(m)}^{\dagger}\right]=\frac{1}{4 g^{2} R^{2}} \boldsymbol{\Sigma}_{(m)}
$$

give the vacua of the Higgs sector of the field theory.

When $k_{0}=k_{1}=\cdots=k_{m}=n$, so that the gauge symmetry restriction is given by

$$
\mathrm{SU}(k) \longrightarrow \mathrm{U}(1)^{m} \times \mathrm{SU}(n)^{m+1} \quad \text { with } \quad k=n(m+1),
$$

an explicit solution of (3.9) is given by $\phi_{(m)}=\phi_{(m)}{ }^{0}$, where

$$
\phi_{i}^{0}=\frac{\zeta_{i}}{2 g R} \sqrt{i(m-i+1)} \mathbf{1}_{n}
$$

for $i=1, \ldots, m$ with $\zeta_{i} \in S^{1}$ independent phase factors. The phases $\zeta_{i}$ can be removed by a $\mathrm{U}(1)^{m}$ gauge transformation in the unbroken symmetry phase. This solution breaks the gauge symmetry of the $d$-dimensional field theory on $M$ to $\mathrm{SU}(n)$. In the broken symmetry phase there are $m n^{2}$ massive gauge bosons, and $m n^{2}$ physical Higgs fields which can be represented in terms of $n \times n$ hermitean matrices $h_{i}, i=1, \ldots, m$ with $\phi_{i}=\phi_{i}^{0}+h_{i}$. The corresponding Higgs masses, proportional to $\frac{1}{R}$, can then be worked out by substitution into the Higgs potential (3.7), while the vector boson masses, also proportional to $\frac{1}{R}$, can be worked out by substitution into the covariant derivative terms of the action (3.6). Note that for $n=1$, the gauge symmetry reduction (3.10) is to the maximal torus of $\mathrm{SU}(m+1)$, and all gauge bosons become massive with no residual gauge symmetry remaining. In the subsequent sections we will look at some explicit examples of such mass generation in the field theory defined by (3.6).

\subsection{Fermionic action for symmetric spinors}

Using the gauged Dirac operator (2.34), we may define a euclidean fermionic action functional on the space of $\mathrm{L}^{2}$-sections of the bundle (2.37) by

$$
S_{\mathrm{D}}:=\int_{M \times \mathbb{C} P^{1}} \mathrm{~d}^{d+2} x \sqrt{|\mathcal{G}|} \Psi^{\dagger} \not \mathcal{D} \Psi
$$

where $\Psi$ has canonical mass dimension $\frac{1}{2}(d+1)$. In lorentzian signature the adjoint spinor $\Psi^{\dagger}$ should be replaced by $\bar{\Psi}:=\frac{1}{\sqrt{-\mathcal{G}^{00}}} \Psi^{\dagger} \Gamma^{0}$. For definiteness, we shall only consider the case where the spinor field $\Psi$ transforms under the fundamental representation of the initial gauge group SU $(k)$. Other fermion representations of $\mathrm{SU}(k)$ can be treated similarly.

One has

$$
\Psi^{\dagger}\left(\gamma\left(\phi_{(m)}\right) \otimes \sigma_{-}+\gamma\left(\phi_{(m)}\right)^{\dagger} \otimes \sigma_{+}\right) \Psi=\left(\begin{array}{ll}
\left(\Psi^{+}\right)^{\dagger} & \left.\left(\Psi^{-}\right)^{\dagger}\right)\left(\begin{array}{c}
\gamma\left(\phi_{(m)}\right)^{\dagger}\left(\Psi^{-}\right) \\
\gamma\left(\phi_{(m)}\right)
\end{array}\left(\Psi^{+}\right)\right.
\end{array}\right) .
$$


Substituting (2.40)-(2.46), we see that (3.13) vanishes on symmetric spinors if $m$ is even. On the other hand, if $m$ is odd there is a surviving contribution from (2.45) and (2.46) when $p_{i}= \pm 1$. For $p_{i}=-1$ the single positive chirality zero mode is a section of the trivial line bundle $\mathcal{L}^{0}$ over $\mathbb{C} P^{1}$, as is the single negative chirality zero mode for $p_{i}=+1$. They are thus globally defined functions on $\mathbb{C} P^{1}$, and hence are simply constants in (2.45) and (2.46) corresponding to $\mathrm{SU}(2)$-singlets in the trivial representation $\underline{V}_{1}$. For these special cases the expression (3.13) produces Yukawa couplings to the fields $\Psi_{(\mp 1)}^{ \pm}$on $M$.

After integration over $\mathbb{C} P^{1}$, and the rescalings $\phi_{i} \rightarrow g R \phi_{i}, \psi_{\left(p_{i}\right) \ell} \rightarrow\left(4 \pi R^{2}\right)^{-1 / 2} \psi_{\left(p_{i}\right) \ell}$, and $\widetilde{\psi}_{\left(p_{i}\right) \ell} \rightarrow\left(4 \pi R^{2}\right)^{-1 / 2} \widetilde{\psi}_{\left(p_{i}\right) \ell}$ to give the scalar and fermion fields the correct canonical dimensions on $M$, the contribution from fermion zero modes on $\mathbb{C} P^{1}$ to the action functional (3.12) is given by

$$
\begin{aligned}
S_{\mathrm{D}}^{0}= & \int_{M} \mathrm{~d}^{d} x \sqrt{|G|}\left[\sum_{i=m_{+}}^{m} \sum_{\ell=0}^{\left|p_{i}\right|-1}\left(\psi_{\left(p_{i}\right) \ell}\right)^{\dagger} \not D\left(\psi_{\left(p_{i}\right) \ell}\right)+\sum_{i=0}^{m_{-}} \sum_{\ell=0}^{\left|p_{i}\right|-1}\left(\widetilde{\psi}_{\left(p_{i}\right) \ell}\right)^{\dagger} \not D\left(\widetilde{\psi}_{\left(p_{i}\right) \ell}\right)\right] \\
& +\frac{g}{2} \int_{M} \mathrm{~d}^{d} x \sqrt{|G|}\left[\left(\psi_{(-1)}\right)^{\dagger} \phi_{m_{+}}^{\dagger} \gamma \widetilde{\psi}_{(1)}+\left(\widetilde{\psi}_{(1)}\right)^{\dagger} \phi_{m_{+}} \gamma \psi_{(-1)}\right]
\end{aligned}
$$

where $m_{-}=\left\lfloor\frac{m-1}{2}\right\rfloor$ and $m_{+}=\left\lceil\frac{m+1}{2}\right\rceil$. The second term in (3.14) is present only when $m$ is odd, in which case $m_{+}=\frac{m+1}{2}$. The fermion fields $\psi_{\left(p_{i}\right) \ell}$ and $\widetilde{\psi}_{\left(p_{i}\right) \ell}$ for each $\ell=0,1, \ldots,\left|p_{i}\right|-1$, with $\psi_{(-1)}:=\psi_{(-1) 0}$ and $\widetilde{\psi}_{(1)}:=\widetilde{\psi}_{(1) 0}$, transform in the fundamental representation of $\mathrm{SU}\left(k_{i}\right)$. Recall that this sector of the field theory on $M$ is induced entirely by the non-trivial monopole background on $\mathbb{C} P^{1}$.

\subsection{Fermionic action for spinor harmonic modes}

For eigenspinors on $\mathbb{C} P^{1}$ with non-zero eigenvalues, the term (3.13) produces an infinite chain of Yukawa couplings to the Higgs fields $\phi_{i}$. After integration over $\mathbb{C} P^{1}$, and again rescaling

$$
\phi_{i} \rightarrow g R \phi_{i}, \quad \psi_{\left(j, p_{i}\right) \ell} \rightarrow\left(4 \pi R^{2}\right)^{-1 / 2} \psi_{\left(j, p_{i}\right) \ell} \quad \text { and } \quad \widetilde{\psi}_{\left(j, p_{i}\right) \ell} \rightarrow\left(4 \pi R^{2}\right)^{-1 / 2} \widetilde{\psi}_{\left(j, p_{i}\right) \ell},
$$

the contributions from non-zero fermion modes on $\mathbb{C} P^{1}$ with positive eigenvalues are given by $\sum_{i=0}^{m} S_{\mathrm{D}}^{\left(p_{i}\right)}$ with

$$
\begin{aligned}
S_{\mathrm{D}}^{\left(p_{i}\right)=} & \int_{M} \mathrm{~d}^{d} x \sqrt{|G|} \sum_{j=j_{\min }}^{\infty} \sum_{\ell=0}^{2 j}\left[\left(\psi_{\left(j, p_{i}\right) \ell}\right)^{\dagger}\left(\not D+\frac{1}{R} \lambda_{j, p_{i}} \gamma\right) \psi_{\left(j, p_{i}\right) \ell}\right. \\
& +\left(\widetilde{\psi}_{\left(j, p_{i}\right) \ell}\right)^{\dagger}\left(\not D+\frac{1}{R} \lambda_{j, p_{i}} \gamma\right) \widetilde{\psi}_{\left(j, p_{i}\right) \ell} \\
& \left.+\frac{g}{2}\left(\psi_{\left(j, p_{i}\right) \ell}\right)^{\dagger} \phi_{i}^{\dagger} \gamma \widetilde{\psi}_{\left(j, p_{i}+2\right) \ell}+\frac{g}{2}\left(\widetilde{\psi}_{\left(j, p_{i}+2\right) \ell}\right)^{\dagger} \phi_{i} \gamma \psi_{\left(j, p_{i}\right) \ell}\right],
\end{aligned}
$$

where $j_{\min }=\max \left(\frac{\left|p_{i}\right|+1}{2}, \frac{\left|p_{i}+2\right|+1}{2}\right)$. Again the fermion fields $\psi_{\left(j, p_{i}\right) \ell}$ and $\widetilde{\psi}_{\left(j, p_{i}\right) \ell}$, for each $j \geq j_{\min }$ and $\ell=0,1, \ldots, 2 j$, transform in the fundamental representation of $\mathrm{SU}\left(k_{i}\right)$.

Consider the truncation of the infinite tower of massive Dirac spinors on the fuzzy sphere $\mathbb{C} P_{F}^{1}$ at $j=j_{\max }$ as described in $\S 2.4$. The fuzzy analogue of the $\mathrm{L}^{2}$-norm $\left\|\chi_{(j, p) \ell}^{ \pm}\right\|_{\mathrm{L}^{2}}$ on $\mathbb{C} P^{1}$ is the matrix trace $\operatorname{Tr}\left[\left(\hat{\chi}_{(j, p) \ell}^{ \pm}\right)^{\dagger} \hat{\chi}_{(j, p) \ell}^{ \pm}\right]$, but observe that $\operatorname{Tr}\left[\left(\hat{\chi}_{(j, p) \ell}^{+}\right)^{\dagger} \hat{\chi}_{\left(j, p^{\prime}\right) \ell}^{-}\right]$is also well-defined if and only if $p^{\prime}=p+2$ and this is exactly what is needed for the Yukawa couplings in (3.16). Note that when $i=0$, the Yukawa terms in (3.16) vanish because there is no fermion field $\widetilde{\psi}_{(j, m+2) \ell}$. Similarly, the minimum value of the monopole charge $p_{i}$ in any spinor $\psi_{\left(j, p_{i}\right)} \ell$ is $-m$, when $i=m$, so 
the fermion fields $\psi_{(j,-m-2) \ell}$ are never present either. Hence $\psi_{(j, m) \ell}$ and $\widetilde{\psi}_{(j,-m) \ell}$ have no Yukawa couplings, because they have no partners to which they can couple.

If the Higgs field $\phi_{i}$ acquires a non-zero vacuum expectation value $\phi_{i}^{0}$ by dynamical symmetry breaking, then the fermion fields $\psi_{\left(j, p_{i}\right) \ell}$ and $\widetilde{\psi}_{\left(j, p_{i}+2\right) \ell}$ acquire a mass matrix. For example, if one takes $k_{0}=k_{1}=\cdots=k_{m}=n$ and $\phi_{i}^{0}=\frac{1}{g R} v_{i} \mathbf{1}_{n}$ as in (3.11), then the mass matrix for each $\ell=0,1, \ldots, 2 j$ is

$$
M_{f}=\frac{1}{R}\left(\begin{array}{cc}
\lambda_{j, p_{i}} & \frac{1}{2} v_{i} \\
\frac{1}{2} \bar{v}_{i} & \lambda_{j, p_{i}+2}
\end{array}\right)
$$

with eigenvalues

$$
\mu_{ \pm}=\frac{1}{2 R}\left(\lambda_{j, p_{i}}+\lambda_{j, p_{i}+2} \pm \sqrt{\left(\lambda_{j, p_{i}}-\lambda_{j, p_{i}+2}\right)^{2}+\left|v_{i}\right|^{2}}\right)
$$

These masses are proportional to $\frac{1}{R}$. In general, it seems plausible that $\mu_{-}$could be very small, or even zero, for specific symmetry breaking patterns, but we have not found an example where this happens. In this example all fermion fields transform in the fundamental representation of the unbroken gauge group $\mathrm{SU}(n)$ after spontaneous symmetry breaking.

Another interesting possibility arises when the metric on $M$ is of lorentzian signature. Then all adjoint spinors $\psi^{\dagger}$ should be replaced by $\bar{\psi}=R \psi^{\dagger} \gamma^{0}$, where the radius factor is necessary to maintain canonical dimensions in our conventions since the gamma-matrix $\gamma^{0}$ has mass dimension one, and similarly for $\widetilde{\psi}^{\dagger}$. With the chiral decompositions $\psi=\psi^{+} \oplus \psi^{-}$and $\widetilde{\psi}=\widetilde{\psi}^{+} \oplus \widetilde{\psi}^{-}$on $M$ satisfying

$$
\gamma \psi^{ \pm}= \pm \psi^{ \pm} \quad \text { and } \quad \gamma \widetilde{\psi}^{ \pm}= \pm \widetilde{\psi}^{ \pm}
$$

we are free to choose $\psi^{-}=\widetilde{\psi}^{+}=0$ for the positive, negative and zero eigenvalues of $D_{\mathbb{C} P^{1}}$. This makes the associated spinors $\Psi_{\left(p_{i}\right)}^{+}, \Psi_{\left(p_{i}\right)}^{-}$, and $\Psi$ all Weyl fermions with positive chirality in $d+2$ dimensions. The direct mass terms involving $\lambda_{j, p_{i}}$ in (3.16) now all vanish leaving only Yukawa interactions in $d$ dimensions.

\section{Dynamical symmetry breaking from the fundamental representation}

We will now work through some explicit, illustrative examples of the dimensionally reduced field theories of $\S 3$. We will obtain the complete physical particle content and compute all fermion masses induced from the dynamical symmetry breaking. In this section we will look at the gauge symmetry decomposition (3.4) in the case of restriction from the lowest non-trivial SU(2) representation, the spin $\frac{1}{2}$ representation. This is the example $m=1$, which corresponds to the fundamental representation of $\mathrm{SU}(2)$. A special instance of this class of examples will involve an electroweak symmetry breaking pattern.

\subsection{Higgs mechanism}

The gauge symmetry reduction is given by $\mathrm{SU}(k) \rightarrow \mathrm{SU}\left(k_{0}\right) \times \mathrm{SU}\left(k_{1}\right) \times \mathrm{U}(1)$ with $k_{0}+k_{1}=k$. The $\mathrm{U}(1)$ factor sits in the fundamental representation of $\mathrm{SU}(k)$ as the generator

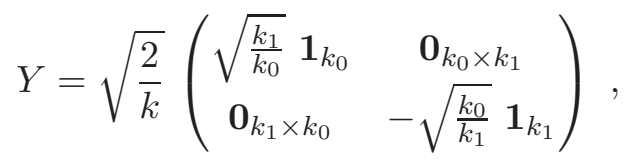

where $\operatorname{tr}_{k \times k}(Y)=0$ and the normalisation is such that $\operatorname{tr}_{k \times k}\left(Y^{2}\right)=2$. Here $\mathbf{0}_{k_{0} \times k_{1}}$ is the $k_{0} \times k_{1}$ zero matrix. 
The U(1) charge of the scalar field $\phi:=\phi_{1}$ follows from (2.27) with $i=0$ and the top left block of $Y$ acting on $\phi$ from the left, as the U(1) part of $A^{0}$, while the bottom right block of $Y$ acts on $\phi$ from the right, as the $\mathrm{U}(1)$ part of $A^{1}$. This gives the $\mathrm{U}(1)$ charge of $\phi$ as

$$
\sqrt{\frac{2}{k}}\left(\sqrt{\frac{k_{1}}{k_{0}}}+\sqrt{\frac{k_{0}}{k_{1}}}\right)=\sqrt{\frac{2 k}{k_{0} k_{1}}} .
$$

The bicovariant derivative $(2.27)$ can be written as

$$
D \phi=\mathrm{d} \phi+\frac{\mathrm{i} g}{2} A_{L}^{a} \lambda_{a} \phi-\frac{\mathrm{i} g}{2} A_{R}^{\tilde{a}} \phi \tilde{\lambda}_{\tilde{a}}+\frac{\mathrm{i} g}{2} \sqrt{\frac{2 k}{k_{0} k_{1}}} B \phi,
$$

where $\lambda_{a}, a=1, \ldots, k_{0}^{2}-1$ and $\tilde{\lambda}_{\tilde{a}}, \tilde{a}=1, \ldots, k_{1}^{2}-1$ are the Gell-Mann matrices for $\mathrm{SU}\left(k_{0}\right)$ and $\mathrm{SU}\left(k_{1}\right)$ respectively, with $A_{L}^{a}$ and $A_{R}^{\tilde{a}}$ the corresponding left and right acting gauge fields, and $B$ is the $\mathrm{U}(1)$ gauge field.

Without loss of generality we shall assume $k_{0} \geq k_{1}$. There is only one Higgs multiplet $\phi$, which is a $k_{0} \times k_{1}$ complex matrix field transforming under $\mathrm{SU}\left(k_{0}\right)$ from the left and $\mathrm{SU}\left(k_{1}\right)$ from the right. The Higgs potential (3.7) becomes

$$
\begin{aligned}
V(\phi) & =\frac{g^{2}}{2} \operatorname{tr}_{k_{0} \times k_{0}}\left(\frac{1}{4 g^{2} R^{2}}-\phi \phi^{\dagger}\right)^{2}+\frac{g^{2}}{2} \operatorname{tr}_{k_{1} \times k_{1}}\left(-\frac{1}{4 g^{2} R^{2}}+\phi^{\dagger} \phi\right)^{2} \\
& =\frac{k_{0}-k_{1}}{32 g^{2} R^{4}}+g^{2} \operatorname{tr}_{k_{1} \times k_{1}}\left(\frac{1}{4 g^{2} R^{2}}-\phi^{\dagger} \phi\right)^{2} .
\end{aligned}
$$

We expect the gauge symmetry to be broken dynamically.

Using the $\mathrm{SU}\left(k_{0}\right) \times \mathrm{SU}\left(k_{1}\right) \times \mathrm{U}(1)$ gauge symmetry, a generic $k_{0} \times k_{1}$ complex matrix $\phi$ can be brought into the form

$$
\phi \longrightarrow U^{(0)} \phi U^{(1)}=\frac{1}{g R}\left(\begin{array}{cccc}
0 & 0 & \cdots & 0 \\
& & \vdots & \\
0 & 0 & \cdots & 0 \\
v_{1} & 0 & \cdots & 0 \\
0 & v_{2} & \cdots & 0 \\
0 & 0 & \cdots & v_{k_{1}}
\end{array}\right)
$$

where $U^{(0)}$ is a $k_{0} \times k_{0}$ unitary matrix, $U^{(1)}$ is a $k_{1} \times k_{1}$ unitary matrix, and $v_{1}, \ldots, v_{k_{1}}$ are non-negative numbers. Putting the form (4.5) into the potential (4.4), we find that the absolute minimum of $V(\phi)$ requires $v_{1}=\cdots=v_{k_{1}}=\frac{1}{2}$. Thus the vacuum expectation value of $\phi$ is a bi-unitary transformation of the matrix

$$
\phi^{0}=\frac{1}{2 g R}\left(\begin{array}{c}
\mathbf{0}_{\left(k_{0}-k_{1}\right) \times k_{1}} \\
\mathbf{1}_{k_{1}}
\end{array}\right) .
$$

The expectation value (4.6) remains invariant under residual $\mathrm{SU}\left(k_{0}-k_{1}\right) \times \mathrm{SU}\left(k_{1}\right)_{\text {diag }} \times \mathrm{U}(1)^{\prime}$ transformations, where $\mathrm{SU}\left(k_{1}\right)_{\text {diag }}$ is the diagonal subgroup of the left and right acting groups $\mathrm{SU}\left(k_{1}\right)_{L} \times \mathrm{SU}\left(k_{1}\right)_{R}$ with $\mathrm{SU}\left(k_{1}\right)_{L}$ acting on the bottom $k_{1}$ rows of $\phi^{0}$, and $\mathrm{U}(1)^{\prime}$ is implemented as acting from the left on the top $k_{0}-k_{1}$ rows of $\phi^{0}$ leaving the bottom $k_{1}$ rows unchanged. This gives the symmetry breaking pattern

$$
\mathrm{SU}\left(k_{0}\right) \times \mathrm{SU}\left(k_{1}\right) \times \mathrm{U}(1) \longrightarrow \mathrm{SU}\left(k_{0}-k_{1}\right) \times \mathrm{SU}\left(k_{1}\right)_{\operatorname{diag}} \times \mathrm{U}(1)^{\prime} .
$$


For the case $k_{1}=1$ the $\mathrm{SU}\left(k_{1}\right)$ factors are omitted.

A total of $2 k_{0} k_{1}-k_{1}^{2}$ gauge bosons acquire masses, proportional to $\frac{1}{R}$, eating up $2 k_{0} k_{1}-k_{1}^{2}$ degrees of freedom from the $k_{0} \times k_{1}$ complex matrix $\phi$ and leaving $k_{1}^{2}$ physical Higgs fields. The latter fields can be arranged into a $k_{1} \times k_{1}$ hermitean matrix $h=h^{\dagger}$ which sits in $\phi$ as

$$
\phi=\left(\begin{array}{c}
\mathbf{0}_{\left(k_{0}-k_{1}\right) \times k_{1}} \\
\frac{1}{2 g R} \mathbf{1}_{k_{1}}+h
\end{array}\right)
$$

with $h$ an $\mathrm{SU}\left(k_{0}-k_{1}\right)$ singlet, transforming as a $k_{1} \times k_{1}$ hermitean matrix under the adjoint action of $\mathrm{SU}\left(k_{1}\right)_{\text {diag }}$, and carrying zero $\mathrm{U}(1)^{\prime}$ charge. Expanding the potential (4.4) in powers of $h$ and examining the quadratic term reveals that the Higgs bosons have mass $\mu_{h}=\frac{1}{R}$.

The precise masses of the gauge bosons can be determined by squaring (2.27) for $i=0$, with $\phi_{1}=\phi^{0}$, and focusing on the part quadratic in the gauge fields. The mass matrix $\boldsymbol{M}$ is a symmetric matrix of dimension $\left(k_{0}^{2}+k_{1}^{2}-1\right) \times\left(k_{0}^{2}+k_{1}^{2}-1\right)$ and of rank $2 k_{0} k_{1}-k_{1}^{2}$ defined via the relation

$$
\frac{1}{2} \boldsymbol{A}^{\top} \boldsymbol{M}^{2} \boldsymbol{A}=g^{2} \operatorname{tr}_{k_{1} \times k_{1}}\left(A^{0} \phi^{0}-\phi^{0} A^{1}\right)^{\dagger}\left(A^{0} \phi^{0}-\phi^{0} A^{1}\right)
$$

where $\boldsymbol{A}$ is a column vector consisting of the $k_{0}^{2}+k_{1}^{2}-1$ gauge bosons in $\mathrm{SU}\left(k_{0}\right) \times \mathrm{SU}\left(k_{1}\right) \times \mathrm{U}(1)$. For example, if $k_{0}=k_{1}=n$, then $\phi^{0}=\frac{1}{2 g R} \mathbf{1}_{n}$ and

$$
\begin{aligned}
\frac{1}{2} \boldsymbol{A}^{\top} \boldsymbol{M}^{2} \boldsymbol{A} & =\frac{g^{2}}{4} \operatorname{tr}\left(A_{L}^{a} \lambda_{a} \phi^{0}-A_{R}^{a} \phi^{0} \lambda_{a}+\frac{2}{\sqrt{n}} B \phi^{0}\right)^{\dagger}\left(A_{L}^{b} \lambda_{b} \phi^{0}-A_{R}^{b} \phi^{0} \lambda_{b}+\frac{2}{\sqrt{n}} B \phi^{0}\right) \\
& =\frac{1}{8 R^{2}}\left(\begin{array}{lll}
A_{L} & A_{R} & B
\end{array}\right)\left(\begin{array}{ccc}
\mathbf{1}_{n^{2}-1} & -\mathbf{1}_{n^{2}-1} & 0 \\
-\mathbf{1}_{n^{2}-1} & \mathbf{1}_{n^{2}-1} & 0 \\
0 & 0 & 2
\end{array}\right)\left(\begin{array}{c}
A_{L} \\
A_{R} \\
B
\end{array}\right)
\end{aligned}
$$

where we have used the normalisation $\operatorname{Tr}\left(\lambda_{a} \lambda_{b}\right)=2 \delta_{a b}$. Diagonalising the mass matrix we find $n^{2}-1$ massless gauge bosons $A^{a}=\frac{1}{\sqrt{2}}\left(A_{L}^{a}+A_{R}^{a}\right)$, and $n^{2}-1$ massive vector bosons $W^{a}=\frac{1}{\sqrt{2}}\left(A_{L}^{a}-A_{R}^{a}\right)$ which, together with the $\mathrm{U}(1)$ gauge boson $B$, all have the same mass $\mu_{W}^{2}=\mu_{B}^{2}=\frac{1}{2 R^{2}}$.

An example which illustrates $\mathrm{U}(1)$ mixing is the case $k_{1}=1$. We start with the simplest instance $k=3$, so that the gauge symmetry reduction is $\mathrm{SU}(3) \rightarrow \mathrm{SU}(2) \times \mathrm{U}(1)$. The Higgs field $\phi$ is a priori a column vector with two complex components. Let $\lambda_{\hat{a}}, \hat{a}=1, \ldots, 8$ be the Gell-Mann matrices generating $\mathrm{SU}(3)$, normalised so that $\operatorname{Tr}\left(\lambda_{\hat{a}} \lambda_{\hat{b}}\right)=2 \delta_{\hat{a} \hat{b}}$. Then the $\mathrm{SU}(2)$ generators can be chosen to be the Pauli spin matrices $\sigma_{a}, a=1,2,3$ with

$$
\lambda_{a}=\left(\begin{array}{cc}
\sigma_{a} & 0 \\
0 & 0
\end{array}\right)
$$

while the $\mathrm{U}(1)$ generator is

$$
\lambda_{8}=\sqrt{\frac{1}{3}}\left(\begin{array}{cc}
\mathbf{1}_{2} & 0 \\
0 & -2
\end{array}\right) .
$$

We thus set

$$
A^{0}=\frac{\mathrm{i}}{2} W^{a} \sigma_{a}+\frac{\mathrm{i}}{2 \sqrt{3}} B \mathbf{1}_{2} \quad \text { and } \quad A^{1}=-\mathrm{i} \sqrt{\frac{1}{3}} B,
$$

where $W^{a}$ are the $\mathrm{SU}(2)$ gauge bosons and $B$ is the $\mathrm{U}(1)$ gauge boson. The gauge coupling to $\phi$ now reads

$$
D \phi=\mathrm{d} \phi+\frac{\mathrm{i} g}{2}\left(W^{a} \sigma_{a}+\sqrt{3} B \mathbf{1}_{2}\right) \phi .
$$


We can use the $\mathrm{SU}(2) \times \mathrm{U}(1)$ gauge symmetry to rotate the vacuum expectation value of $\phi$ to

$$
\phi^{0}=\frac{1}{2 g R}\left(\begin{array}{l}
0 \\
1
\end{array}\right)
$$

and the quadratic form (4.9) becomes

$$
\frac{1}{2}\left(\begin{array}{ll}
\boldsymbol{W}^{\top} & B
\end{array}\right) \boldsymbol{M}^{2}\left(\begin{array}{c}
\boldsymbol{W} \\
B
\end{array}\right)=\frac{1}{16 R^{2}}\left(W^{a} W^{b} \delta_{a b}+3 B^{2}-2 \sqrt{3} W^{3} B\right)
$$

with $\boldsymbol{W}$ a three-component column vector. From this equation we can read off the $4 \times 4$ mass matrix

$$
M^{2}=\frac{1}{8 R^{2}}\left(\begin{array}{cccc}
1 & 0 & 0 & 0 \\
0 & 1 & 0 & 0 \\
0 & 0 & 1 & -\sqrt{3} \\
0 & 0 & -\sqrt{3} & 3
\end{array}\right)
$$

The photon, i.e. the $\mathrm{U}(1)^{\prime}$ gauge boson, is the massless combination

$$
A=\frac{1}{2}\left(B+\sqrt{3} W^{3}\right),
$$

while the $W$-bosons acquire a mass $\mu_{W^{ \pm}}=\frac{1}{2 \sqrt{2} R}$ and the $Z$-boson a mass $\mu_{Z}=2 \mu_{W^{ \pm}}=\frac{1}{\sqrt{2} R}$. Clearly this is not a realistic model for electroweak interactions, since the Weinberg angle is too large at $\sin ^{2} \theta_{\mathrm{W}}=\frac{3}{4}$. Nevertheless, it is an instructive example for the symmetry hierarchy given by $\mathrm{SU}(3) \rightarrow \mathrm{SU}(2) \times \mathrm{U}(1) \rightarrow \mathrm{U}(1)$ in this class of models.

A similar analysis can be carried through for $k_{0}>2$ and $k_{1}=1$. In this case $2 k_{0}-1$ of the $\mathrm{SU}\left(k_{0}\right)$ gauge bosons acquire a mass while $k_{0}^{2}-2 k_{0}$ of them remain massless, and the residual gauge symmetry is $\mathrm{SU}\left(k_{0}-1\right)$. There are $2 k_{0}-1 W$-bosons, one of which mixes with $B$ to form a massive $Z$-boson while leaving an orthogonal linear combination massless. The mass matrix is of the form

$$
\boldsymbol{M}^{2}=\frac{1}{8 R^{2}}\left(\begin{array}{cccc}
\mathbf{0}_{k_{0}\left(k_{0}-2\right) \times k_{0}\left(k_{0}-2\right)} & 0 & \cdots & 0 \\
0 & \mathbf{1}_{2\left(k_{0}-1\right)} & 0 & 0 \\
\vdots & 0 & \frac{2\left(k_{0}-1\right)}{k_{0}} & -\frac{2 \sqrt{k_{0}^{2}-1}}{k_{0}} \\
0 & 0 & -\frac{2 \sqrt{k_{0}^{2}-1}}{k_{0}} & \frac{2\left(k_{0}+1\right)}{k_{0}}
\end{array}\right) .
$$

The gauge boson masses are independent of $k_{0}$. The $W$-boson mass is $\mu_{W}=\frac{1}{2 \sqrt{2} R}$, while diagonalising the bottom right $2 \times 2$ matrix block again reveals a $Z$-boson mass $\mu_{Z}=\frac{1}{\sqrt{2} R}$. The same quantitative features hold for any value of $k_{0}$ in the symmetry reduction sequence

$$
\mathrm{SU}\left(2 k_{0}\right) \longrightarrow \mathrm{SU}\left(k_{0}\right) \times \mathrm{SU}\left(k_{0}\right) \times \mathrm{U}(1) \longrightarrow \mathrm{SU}\left(k_{0}\right)_{\operatorname{diag}} \times \mathrm{U}(1)^{\prime}
$$

with the ground state of the Higgs sector given by (3.11). In all instances we get the mass hierarchy

$$
\mu_{h}=\sqrt{2} \mu_{Z}=2 \sqrt{2} \mu_{W}=\frac{1}{R}
$$

\subsection{Yukawa interactions}

The $d$-dimensional fermion fields in this model are

$$
\widetilde{\psi}_{(1)}, \quad \psi_{(j, 1) \ell}, \quad \text { and } \quad \widetilde{\psi}_{(j, 1) \ell}
$$


in the fundamental representation of $\mathrm{SU}\left(k_{0}\right)$, and

$$
\psi_{(-1)}, \quad \psi_{(j,-1) \ell}, \quad \text { and } \quad \widetilde{\psi}_{(j,-1) \ell}
$$

in the fundamental representation of $\mathrm{SU}\left(k_{1}\right)$. In both cases $j \geq 1$ and $\ell=0,1, \ldots, 2 j$. The Yukawa couplings are obtained from (3.14) and (3.16), with $m=1$ and $i=0,1$. After the rescalings (3.15), they are given by

$$
S_{\mathrm{Y}}^{(j, \ell)}=\frac{g}{2} \int_{M} \mathrm{~d}^{d} x \sqrt{|G|}\left[\left(\psi_{(j,-1) \ell}\right)^{\dagger} \phi^{\dagger} \gamma \widetilde{\psi}_{(j, 1) \ell}+\left(\widetilde{\psi}_{(j, 1) \ell}\right)^{\dagger} \phi \gamma \psi_{(j,-1) \ell}\right]
$$

from the Dirac eigenspinors on $\mathbb{C} P^{1}$, and

$$
S_{\mathrm{Y}}^{0}=\frac{g}{2} \int_{M} \mathrm{~d}^{d} x \sqrt{|G|}\left[\left(\psi_{(-1)}\right)^{\dagger} \phi^{\dagger} \gamma \widetilde{\psi}_{(1)}+\left(\widetilde{\psi}_{(1)}\right)^{\dagger} \phi \gamma \psi_{(-1)}\right]
$$

from the zero modes.

When the Higgs field $\phi$ is of the form (4.8), the gauge symmetry is broken as in (4.7), and the Yukawa couplings for the spinors (4.22) and (4.23) are given by

$$
\begin{aligned}
S_{\mathrm{Y}}= & \frac{1}{4 R} \int_{M} \mathrm{~d}^{d} x \sqrt{|G|}\left[\left(\psi_{(j,-1) \ell}\right)^{\dagger} \gamma \widetilde{\psi}_{(j, 1) \ell}+\left(\psi_{(-1)}\right)^{\dagger} \gamma \widetilde{\psi}_{(1)}\right] \\
& +\frac{g}{2} \int_{M} \mathrm{~d}^{d} x \sqrt{|G|}\left[\left(\psi_{(j,-1) \ell}\right)^{\dagger} h \gamma \widetilde{\psi}_{(j, 1) \ell}+\left(\psi_{(-1)}\right)^{\dagger} h \gamma \widetilde{\psi}_{(1)}\right]+\text { h.c. }
\end{aligned}
$$

All fermion fields transform in the fundamental representation of $\mathrm{SU}\left(k_{1}\right)_{\text {diag }}$ after spontaneous symmetry breaking. There are no Yukawa couplings for any of the fermion fields $\psi_{(j, 1)}$ or $\widetilde{\psi}_{(j,-1)}$, although they pick up direct mass terms proportional to $\frac{1}{R}$ from the eigenvalues $\lambda_{j, \pm 1}=\sqrt{j(j+1)}$. The mass matrix for the fermions $\psi_{(j,-1)}$ and $\widetilde{\psi}_{(j, 1)}$ has eigenvalues

$$
\mu_{ \pm}=\frac{1}{R}\left(\sqrt{j(j+1)} \pm \frac{1}{4}\right)
$$

For the fuzzy sphere truncation at $j_{\max }=0$, all spinors $\psi_{(j, \pm 1) \ell}$ and $\widetilde{\psi}_{(j, \pm 1) \ell}$ vanish and only the zero modes survive, leaving two flavours of Dirac fermions in $d$ dimensions, $\psi_{(-1)}$ and $\widetilde{\psi}_{(1)}$, with Yukawa couplings and masses $\frac{1}{4 R}$ but no direct mass term.

\section{Dynamical symmetry breaking from the adjoint representation}

Our final example corresponds to the equivariant gauge theory which descends from the spin one, adjoint representation of $\mathrm{SU}(2)$, having $m=2$. This example constitutes the simplest case in which the symmetry breaking is determined by a chain of Higgs multiplets. As in the previous section, we shall compute the complete physical excitation spectrum in both the bosonic and fermionic sectors for the spontaneously broken symmetry phase of the field theory.

\subsection{Higgs mechanism}

Let us consider the case in which $k=3 n$ with $k_{0}=k_{1}=k_{2}=n$. Then the gauge symmetry reduction sequence is

$$
\mathrm{SU}(3 n) \longrightarrow \mathrm{SU}(n)_{1} \times \mathrm{SU}(n)_{2} \times \mathrm{SU}(n)_{3} \times \mathrm{U}(1) \times \mathrm{U}(1)^{\prime}
$$


There are two complex $n \times n$ matrices of Higgs fields $\phi_{1}$, with $\mathrm{SU}(n)_{1}$ acting on the left and $\mathrm{SU}(n)_{2}$ acting on the right, and $\phi_{2}$, with $\mathrm{SU}(n)_{2}$ acting on the left and $\mathrm{SU}(n)_{3}$ on the right. The $\mathrm{U}(1)$ and $\mathrm{U}(1)^{\prime}$ generators can be taken to be any two linearly independent generators of $\mathrm{SU}(3 n)$ which commute with all three $\mathrm{SU}(n)$ factors. In particular, we can take

$$
Y=\frac{1}{\sqrt{n}}\left(\begin{array}{ccc}
\mathbf{1} & \mathbf{0} & \mathbf{0} \\
\mathbf{0} & -\mathbf{1} & \mathbf{0} \\
\mathbf{0} & \mathbf{0} & \mathbf{0}
\end{array}\right) \quad \text { and } \quad Y^{\prime}=\frac{1}{\sqrt{n}}\left(\begin{array}{ccc}
\mathbf{0} & \mathbf{0} & \mathbf{0} \\
\mathbf{0} & \mathbf{1} & \mathbf{0} \\
\mathbf{0} & \mathbf{0} & -\mathbf{1}
\end{array}\right)
$$

where each entry is an $n \times n$ matrix. With this normalisation the scalar field $\phi_{1}$ has $Y$-charge $\frac{2}{\sqrt{n}}$ and $Y^{\prime}$-charge $-\frac{1}{\sqrt{n}}$, while $\phi_{2}$ has $Y$-charge $-\frac{1}{\sqrt{n}}$ and $Y^{\prime}$-charge $\frac{2}{\sqrt{n}}$.

The Higgs potential (3.7) is

$$
V\left(\phi_{1}, \phi_{2}\right)=\frac{n}{4 g^{2} R^{4}}-\frac{1}{2 R^{2}} \operatorname{tr}\left(\phi_{1}^{\dagger} \phi_{1}+\phi_{2}^{\dagger} \phi_{2}\right)+g^{2} \operatorname{tr}\left(\left(\phi_{1}^{\dagger} \phi_{1}\right)^{2}-\left(\phi_{1}^{\dagger} \phi_{1} \phi_{2}^{\dagger} \phi_{2}\right)+\left(\phi_{2}^{\dagger} \phi_{2}\right)^{2}\right) \text {. }
$$

The general solution (3.11) in this case, with $\zeta_{1}=\zeta_{2}=1$, gives the vacuum configuration

$$
\phi_{1}^{0}=\phi_{2}^{0}=\frac{1}{\sqrt{2} g R} \mathbf{1}_{n} .
$$

This expectation value is invariant under a single copy of $\mathrm{SU}(n)$, which is a linear combination of $\mathrm{SU}(n)_{1}, \mathrm{SU}(n)_{2}$ and $\mathrm{SU}(n)_{3}$. There is no linear combination of $\mathrm{U}(1)$ and $\mathrm{U}(1)^{\prime}$ that leaves it invariant, so the gauge symmetry is reduced as

$$
\mathrm{SU}(3 n) \longrightarrow \mathrm{SU}(n)_{1} \times \mathrm{SU}(n)_{2} \times \mathrm{SU}(n)_{3} \times \mathrm{U}(1) \times \mathrm{U}(1)^{\prime} \longrightarrow \mathrm{SU}(n) .
$$

In this case $2 n^{2}$ gauge bosons acquire a mass and, of the $4 n^{2}$ degrees of freedom in $\phi_{1}$ and $\phi_{2}$, a total of $2 n^{2}$ remain as physical Higgs fields. Expanding around the ground state, the latter fields can be represented by $n \times n$ hermitean matrices $h_{1}$ and $h_{2}$ with

$$
\phi_{1}=\frac{1}{\sqrt{2} g R} \mathbf{1}_{n}+h_{1} \quad \text { and } \quad \phi_{2}=\frac{1}{\sqrt{2} g R} \mathbf{1}_{n}+h_{2} .
$$

Putting this form into (5.3) and examining the terms quadratic in $h_{1}$ and $h_{2}$, we find a $2 \times 2$ mass matrix with Higgs masses given by the eigenvalues

$$
\mu_{h_{>}}=\frac{\sqrt{3}}{R} \quad \text { and } \quad \mu_{h_{<}}=\frac{1}{R} .
$$

The vector boson masses are derived by diagonalising the $\left(3 n^{2}-1\right) \times\left(3 n^{2}-1\right)$ mass matrix arising from the identity

$$
\begin{aligned}
& \frac{1}{2} \boldsymbol{A}^{\top} \boldsymbol{M}^{2} \boldsymbol{A} \\
& =\frac{1}{8 R^{2}} \operatorname{Tr}\left[\left(\left(A_{1}^{a}-A_{2}^{a}\right) \lambda_{a}+\frac{2}{\sqrt{n}} B \mathbf{1}_{n}-\frac{1}{\sqrt{n}} B^{\prime} \mathbf{1}_{n}\right)^{\dagger}\left(\left(A_{1}^{b}-A_{2}^{b}\right) \lambda_{b}+\frac{2}{\sqrt{n}} B \mathbf{1}_{n}-\frac{1}{\sqrt{n}} B^{\prime} \mathbf{1}_{n}\right)\right. \\
& \left.\quad+\left(\left(A_{2}^{a}-A_{3}^{a}\right) \lambda_{a}-\frac{1}{\sqrt{n}} B \mathbf{1}_{n}+\frac{2}{\sqrt{n}} B^{\prime} \mathbf{1}_{n}\right)^{\dagger}\left(\left(A_{1}^{b}-A_{2}^{b}\right) \lambda_{b}-\frac{1}{\sqrt{n}} B \mathbf{1}_{n}+\frac{2}{\sqrt{n}} B^{\prime} \mathbf{1}_{n}\right)\right]
\end{aligned}
$$

where $B$ and $B^{\prime}$ are the gauge fields associated with $\mathrm{U}(1)$ and $\mathrm{U}(1)^{\prime}$ respectively. This gives

$$
\boldsymbol{M}^{2}=\frac{1}{4 R^{2}}\left(\begin{array}{ccccc}
2 \mathbf{1} & -2 \mathbf{1} & \mathbf{0} & 0 & 0 \\
-2 \mathbf{1} & 4 \mathbf{1} & -2 \mathbf{1} & 0 & 0 \\
\mathbf{0} & -2 \mathbf{1} & 2 \mathbf{1} & 0 & 0 \\
0 & 0 & 0 & 5 & -4 \\
0 & 0 & 0 & -4 & 5
\end{array}\right)
$$


where each bold-face matrix block is of dimension $\left(n^{2}-1\right) \times\left(n^{2}-1\right)$. The massless $\mathrm{SU}(n)$ gauge bosons are

$$
A^{a}=\frac{1}{\sqrt{3}}\left(A_{1}^{a}+A_{2}^{a}+A_{3}^{a}\right),
$$

while the vector bosons $W^{a}=\frac{1}{\sqrt{2}}\left(A_{1}^{a}-A_{3}^{a}\right)$ acquire a mass

$$
\mu_{W}=\frac{1}{\sqrt{2} R}
$$

and $V^{a}=\frac{1}{\sqrt{6}}\left(A_{1}^{a}-2 A_{2}^{a}+A_{3}^{a}\right)$ have mass

$$
\mu_{V}=\sqrt{\frac{3}{2}} \frac{1}{R}
$$

The U(1) vector bosons mix as

$$
Z=\frac{1}{\sqrt{2}}\left(B+B^{\prime}\right) \quad \text { and } \quad Z^{\prime}=\frac{1}{\sqrt{2}}\left(B-B^{\prime}\right),
$$

with masses

$$
\mu_{Z}=\frac{1}{2 R} \quad \text { and } \quad \mu_{Z^{\prime}}=\frac{3}{2} \frac{1}{R} .
$$

\subsection{Yukawa interactions}

The $d$-dimensional fermion fields in this model are

$$
\widetilde{\psi}_{(2)}, \quad \psi_{(j, 2) \ell}, \quad \text { and } \quad \widetilde{\psi}_{(j, 2) \ell} \quad \text { with } \quad j \geq \frac{3}{2}
$$

in the fundamental representation of $\mathrm{SU}(n)_{1}$, together with

$$
\psi_{(j, 0) \ell} \quad \text { and } \quad \widetilde{\psi}_{(j, 0) \ell} \quad \text { with } \quad j \geq \frac{1}{2}
$$

in the fundamental representation of $\mathrm{SU}(n)_{2}$, and

$$
\psi_{(-2)}, \quad \psi_{(j,-2) \ell}, \quad \text { and } \quad \widetilde{\psi}_{(j,-2) \ell} \quad \text { with } j \geq \frac{3}{2}
$$

in the fundamental representation of $\mathrm{SU}(n)_{3}$. In all cases $\ell=0,1, \ldots, 2 j$. The Yukawa couplings after dimensional reduction are

$$
S_{\mathrm{Y}}=\frac{g}{2} \int_{M} \mathrm{~d}^{d} x \sqrt{|G|}\left[\left(\widetilde{\psi}_{(j, 2) \ell}\right)^{\dagger} \phi_{1} \psi_{(j, 0) \ell}+\left(\widetilde{\psi}_{(j, 0) \ell}\right)^{\dagger} \phi_{2} \psi_{(j,-2) \ell}\right]+\text { h.c. }
$$

as the spinor fields $\psi_{(-2)}, \widetilde{\psi}_{(2)}, \psi_{(j, 2) \ell}$ and $\widetilde{\psi}_{(j,-2) \ell}$ have no Yukawa couplings. The former two fermions are massless, while the latter two fermions have direct mass terms proportional to $\frac{1}{R}$ coming from the eigenvalues $\lambda_{j, \pm 2}=\sqrt{\left(j-\frac{1}{2}\right)\left(j+\frac{3}{2}\right)}$. All fermion fields transform in the fundamental representation of $\mathrm{SU}(n)$ after spontaneous symmetry breaking. After substituting the Higgs fields (5.6) into (5.18), the Yukawa masses can be read off from the general formula (3.18) with $p_{i}=0$ and $\left|v_{i}\right|^{2}=\frac{1}{2}$. 


\section{Conclusions}

In this paper we have explicitly worked out the $\mathrm{SU}(2)$-equivariant dimensional reduction of pure massless Yang-Mills-Dirac theory over the coset space $\mathbb{C} P^{1}$, including a systematic incorporation of Dirac monopole backgrounds. The internal magnetic fluxes induce a Higgs potential as well as Yukawa couplings between the reduced fermion fields and the Higgs fields, with the standard form of dynamical symmetry breaking. In particular, in certain instances the zero modes of the Dirac operator on $\mathbb{C} P^{1}$ acquire Yukawa interactions. In our formulation we are able to naturally induce both massive and massless fermions, as well as a chiral gauge theory. When inducing massive Dirac spinors associated to higher spinor harmonics on $\mathbb{C} P^{1}$, it is more natural to use a fuzzy sphere $\mathbb{C} P_{F}^{1}$ for the internal space, as it provides an $\mathrm{SU}(2)$-equivariant truncation of the infinite tower of modes and can also be used to truncate to the finitely-many flavours of massless symmetric spinor modes. We worked out several explicit examples of spontaneous symmetry breaking, including classes containing the standard electroweak symmetry breaking sequence as a special case and also a class involving a chain of Higgs fields. In all cases we explicitly worked out the complete physical particle spectrum in the dimensionally reduced field theory after dynamical symmetry breaking.

There are a few technical points which we have brushed over in our analysis. For example, we have not analysed the stability of the Higgs vacua $\phi_{i}^{0}$ that led to dynamical symmetry breaking. Although the spectrum of fluctuations around the solutions we have used certainly do not contain any unstable modes, because these vacua minimize the Higgs potential, one should check whether or not there are any flat modes which may lead to a non-trivial vacuum moduli space. This appears to be a rather non-trivial task even for the simplest Higgs vacua we have found. We have also not addressed the problem of renormalizability of the dimensionally reduced field theory. Since the original higher-dimensional Yang-Mills-Dirac theory is generically non-renormalizable, keeping all higher modes in the lower-dimensional model generically leads to a non-renormalizable field theory. It is not clear if the truncations we have used can help to give better quantum behaviour. It would be interesting to analyse further if any symmetries of the coupled chain field system (e.g. supersymmetry) could lead to renormalizable quantum field theories after dimensional reduction.

In this article we have only focused on the simplest possible homogeneous space to elucidate as clearly as possible the effects of topologically non-trivial gauge field configurations obtained by gauging the holonomy group of the coset. In principle, one can consider more complicated coset spaces $G / H$ with the hope of obtaining more realistic physical theories resembling the standard model. As regards the fermionic sector, a particularly crucial role is played by those cosets which admit a finite-dimensional matrix approximation $(G / H)_{F}$, such as the fuzzy complex projective spaces $\mathbb{C} P_{F}^{N}$ where an explicit universal Dirac operator is known and whose spectrum has been studied in detail in [22]. For $N=2$, the $\mathrm{SU}(3)$-equivariant dimensional reduction of Yang-Mills theory over $\mathbb{C} P^{2}$ has been carried out in detail in [14] incorporating both $\mathrm{SU}(2)$ instanton and $\mathrm{U}(1)$ monopole backgrounds associated with the holonomy group $\mathrm{U}(3)$ of $\mathbb{C} P^{2}$. It would be interesting to extend the techniques of this paper to these classes of equivariant dimensional reduction schemes. In particular, one can compare with results of [6] where the use of (fuzzy) complex projective planes has been suggested as a natural internal space for Kaluza-Klein reduction, leading to the appropriate chiral fermionic spectrum of the standard model.

It would also be interesting to use our techniques to study the reductions of the ten-dimensional $\mathcal{N}=1$ supersymmetric $\mathrm{E}_{8}$ gauge theories over six-dimensional coset spaces considered in [2, 3], although many of these cosets have no known fuzzy versions. Nevertheless, the SU(3) equivariant dimensional reduction of Yang-Mills theory over the six-dimensional non-symmetric space $\mathrm{SU}(3) / \mathrm{U}(1)^{2}$ is explicitly worked out in [14] including $\mathrm{U}(1)$ monopole backgrounds associated with the maximal torus $\mathrm{U}(1) \times \mathrm{U}(1)$ of $\mathrm{SU}(3)$. An outline of a scheme that could allow for a fuzzy version of this coset space was proposed in [23]. It would be interesting to compare the resulting 
four-dimensional field theories with those of [3], particularly the supersymmetry properties which arise under equivariant dimensional reduction. Our reduction techniques could also be applied in principle to the superstring theories on nearly Kähler backgrounds considered in [4]. In this regard it would be interesting to find a natural interpretation for the internal fluxes within the context of these superstring models, along the lines of the flux stabilization mechanisms on arrays of D-branes in Type II string theory suggested in $[8,11,12,14]$.

\section{Acknowledgments}

B.P.D. wishes to thank the Dublin Institute of Advanced Studies for financial support. The work of R.J.S. was supported in part by the EU-RTN Network Grant MRTN-CT-2004-005104.

\section{References}

[1] P. Forgács and N.S. Manton, Commun. Math. Phys. 72 (1980) 15; C.H. Taubes, Commun. Math. Phys. 75 (1980) 207.

[2] D. Kapetanakis and G. Zoupanos, Phys. Rept. 219 (1992) 1.

[3] P. Manousselis and G. Zoupanos, JHEP 03 (2002) 002 [arXiv:hep-ph/0111125]; JHEP 11 (2004) 025 [arXiv:hep-ph/0406207]; A. Chatzistavrakidis, P. Manousselis, N. Prezas and G. Zoupanos, Phys. Lett. B 656 (2007) 152 [arXiv:0708.3222 [hep-th]]; G. Douzas, T. Grammatikopoulos and G. Zoupanos, arXiv:0808.3236 [hep-th].

[4] G. Lopes Cardoso, G. Curio, G. Dall'Agata, D. Lüst, P. Manousselis and G. Zoupanos, Nucl. Phys. B 652 (2003) 5 [arXiv:hep-th/0211118]; P. Manousselis, N. Prezas and G. Zoupanos, Nucl. Phys. B 739 (2006) 85 [arXiv:hep-th/0511122]; A. Chatzistavrakidis, P. Manousselis and G. Zoupanos, arXiv:0811.2182 [hep-th].

[5] E. Witten, in: Proceedings of the 1983 Shelter Island Conference on Quantum Field Theory and the Fundamental Problems of Physics, eds. R. Jackiw, N.N. Khuri, S. Weinberg and E. Witten (MIT Press, 1985).

[6] B.P. Dolan and C. Nash, JHEP 10 (2002) 041 [arXiv:hep-th/0207078]; JHEP 07 (2002) 057 [arXiv:hep-th/0207007].

[7] L. Álvarez-Cónsul and O. García-Prada, J. Reine Angew. Math. 556 (2003) 1 [arXiv:math.DG/0112160]; Commun. Math. Phys. 238 (2003) 1 [arXiv:math.DG/0112161].

[8] O. Lechtenfeld, A.D. Popov and R.J. Szabo, Progr. Theor. Phys. Suppl. 171 (2007) 258 [arXiv:0706.0979 [hep-th]].

[9] O. García-Prada, Commun. Math. Phys. 156 (1993) 527; Int. J. Math. 5 (1994) 1.

[10] L. Álvarez-Cónsul and O. García-Prada, Int. J. Math. 12 (2001) 159.

[11] O. Lechtenfeld, A.D. Popov and R.J. Szabo, JHEP 12 (2003) 022 [arXiv:hep-th/0310267]; JHEP 09 (2006) 054 [arXiv:hep-th/0603232].

[12] A.D. Popov and R.J. Szabo, J. Math. Phys. 47 (2006) 012306 [arXiv:hep-th/0504025].

[13] A.D. Popov, arXiv:0712.1756 [hep-th]; Lett. Math. Phys. 84 (2008) 139 [arXiv:0801.0808 [hepth]]. 
[14] O. Lechtenfeld, A.D. Popov and R.J. Szabo, JHEP 08 (2008) 093 [arXiv:0806.2791 [hep-th]].

[15] A.D. Popov, Phys. Rev. D 77 (2008) 125026 [arXiv:0803.3320 [hep-th]]; arXiv:0804.3845 [hepth].

[16] G.B. Segal, Publ. Math. IHES (Paris) 34 (1968) 113; 129.

[17] M. Cahen, A. Franc and S. Gutt, Lett. Math. Phys. 32 (1994) 365; C. Bär, Analysis 6 (1996) 899.

[18] J. Madore, Class. Quant. Grav. 9 (1992) 69.

[19] P. Aschieri, J. Madore, P. Manousselis and G. Zoupanos, JHEP 04 (2004) 034 [arXiv:hepth/0310072].

[20] P. Aschieri, T. Grammatikopoulos, H. Steinacker and G. Zoupanos, JHEP 09 (2006) 026 [arXiv:hep-th/0606021]; H. Steinacker and G. Zoupanos, JHEP 09 (2007) 017 [arXiv:0706.0398 [hep-th]].

[21] H. Grosse and P. Presnajder, Lett. Math. Phys. 33 (1995) 171; H. Grosse, C. Klimcik and P. Presnajder, Commun. Math. Phys. 178 (1996) 507 [arXiv:hep-th/9510083]; U. CarowWatamura and S. Watamura, Commun. Math. Phys. 183 (1997) 365 [arXiv:hep-th/9605003].

[22] B.P. Dolan, I. Huet, S. Murray and D. O'Connor, JHEP 03 (2008) 029 [arXiv:0711.1347 [hepth]].

[23] C. Sämann, JHEP 02 (2008) 111 [arXiv:hep-th/0612173]. 